\title{
Electrochemical Dearomatization of Commodity Polymers
}

\section{Zohaib Siddiqi and David Sarlah*}

Roger Adams Laboratory, Department of Chemistry, University of Illinois, Urbana, Illinois 61801, United States sarlah@illinois.edu

\section{Supporting Information}




\section{TABLE OF CONTENTS}

1. General Experimental S3

2. Experimental Set-up S4

2-1. Small scale reactions $(<4.0 \mathrm{mmol}$ scale) $\quad \mathrm{S} 4$

2-2. Large scale reactions (>50 mmol) S4

3. Experimental Procedures $\quad$ S5

3-1. Initial screening of viable poly(styrene) reduction conditions $\quad$ S5

3-2. Optimized reaction conditions and selected optimization entries $\quad$ S5

3-3. General procedure for the dearomatization of polyarenes S6

3-4. Failed Substrates $\quad$ S28

3-5. Large Scale Procedures $\quad$ S29

3-6. Different Mw Experiment S31

3-7. Derivatization $\quad$ S32

4. References $\quad$ S39 


\section{General Experimental}

Unless otherwise noted, all reactions were carried out under inert atmosphere $\left(\mathrm{N}_{2}\right)$. Hexamethylphosphoramide (HMPA), ${ }^{1}$ lithium bromide, ${ }^{2}$ and dimethylurea (DMU) ${ }^{2}$ were purified according to the literature procedure. Tetrahydrofuran (HPLC grade), dichloromethane (ACS grade), and toluene (ACS grade) were dried using an MB-SPS solvent purification system containing activated alumina manufactured by MBRAUN. Monodisperse $17 \mathrm{kDa}$ poly(styrene) was prepared according to the literature procedure $^{3}$ Other monodisperse poly(styrene) samples were obtained from commercial vendors. Commercial polymers were purified through two methanol precipitations from dichloromethane and dried under vacuum overnight at room temperature. Poly(styrene-co-allyl-alcohol) was used as received. All other chemicals were purchased from commercial vendors and used without further purification.

${ }^{1} \mathrm{H}$ and ${ }^{13} \mathrm{C}$ NMR spectra were recorded on Varian Unity $500\left(500 \mathrm{MHz},{ }^{1} \mathrm{H} ; 126 \mathrm{MHz},{ }^{13} \mathrm{C}\right)$ or Bruker 500 $\left(500 \mathrm{MHz},{ }^{1} \mathrm{H} ; 126 \mathrm{MHz},{ }^{13} \mathrm{C}\right)$ or Bruker $600\left(500 \mathrm{MHz},{ }^{1} \mathrm{H} ; 151 \mathrm{MHz},{ }^{13} \mathrm{C}\right)$ spectrometers. Spectra are referenced to residual chloroform $\left(\delta=7.26 \mathrm{ppm},{ }^{1} \mathrm{H} ; 77.16 \mathrm{ppm},{ }^{13} \mathrm{C}\right)$. Chemical shifts are reported in parts per million (ppm).

Differential scanning calorimetry (DSC) was performed using a TA Q2500. Samples were prepared in Tzero pans and generally analyzed from $40^{\circ} \mathrm{C}$ to $150^{\circ} \mathrm{C}$. In the case of 5-7, temperature ranges of $-100^{\circ} \mathrm{C}$ to $150{ }^{\circ} \mathrm{C}$ were utilized. In the case of 15,16 , temperature ranges of $40^{\circ} \mathrm{C}$ to $180^{\circ} \mathrm{C}$ were utilized. In all cases a heating rate of $10^{\circ} \mathrm{C} / \mathrm{min}$. was implemented, and data was analyzed from the second heating cycle. Thermal gradient analysis (TGA) was performed using a TA Q50 from $40^{\circ} \mathrm{C}-600{ }^{\circ} \mathrm{C}$ at a heating rate of $15^{\circ} \mathrm{C} / \mathrm{min}$. The degradation point was defined as the onset point for all experiments. Infrared spectra were measured neat on a Perkin-Elmer spectrum BX FT-ATR IR spectrometer.

Gel permeation chromatography (GPC) was performed using a Tosoh Ecosec HLC-8320GPC at $40{ }^{\circ} \mathrm{C}$ fitted with a reference column $(6.0 \mathrm{~mm}$ ID $\times 15 \mathrm{~cm})$, a guard column $(6.0 \mathrm{~mm} \mathrm{ID} \times 4.0 \mathrm{~cm} \times 5 \mu \mathrm{m})$, and two analytical columns $(7.8 \mathrm{~mm}$ ID $\times 30 \mathrm{~cm} \times 5 \mu \mathrm{m})$. The reference flow rate is $0.5 \mathrm{~mL} / \mathrm{min}$ while the analytical column is at $1.0 \mathrm{~mL} / \mathrm{min}$. THF was used as the eluent, and poly(styrene) standards (15 points ranging from $500 \mathrm{Mw}$ to 8.42 million $\mathrm{Da}$ ) were used as the general calibration. 


\section{Experimental Set-up}

\section{2-1. Small scale reactions ( $<4.0 \mathrm{mmol}$ scale)}

Vial: Machery-Nagel item no. 702023

Cap: Machery-Nagel item no. 702060

Septa: Machery-Nagel item no. 702062

Cathode: Magnesium Alloy AZ31B - cut into $3.0 \mathrm{~mm} \times 1.0 \mathrm{~cm} \times 5.0 \mathrm{~cm}$ rectangles

Mounted using a toothless alligator clip attached to a copper wire

Anode: 22-gauge galvanized steel wire

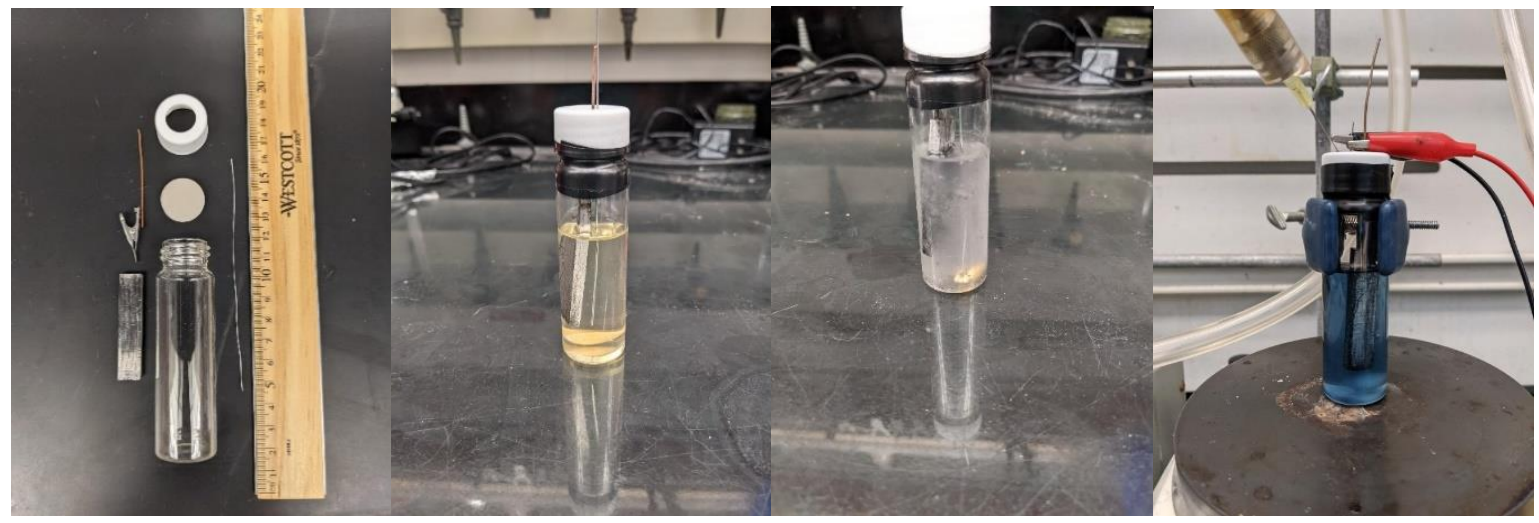

Figure S1. Left: Disassembled undivided electrochemical cell. Left Middle: Fully assembled cell. Right Middle: Reaction after completion. Right: The blue color was observed after 2.0F/mol (commercial PS) or complete conversion (low MW, monodisperse PS).

\section{2-2. Large scale reactions $(>50 \mathrm{mmol})$}

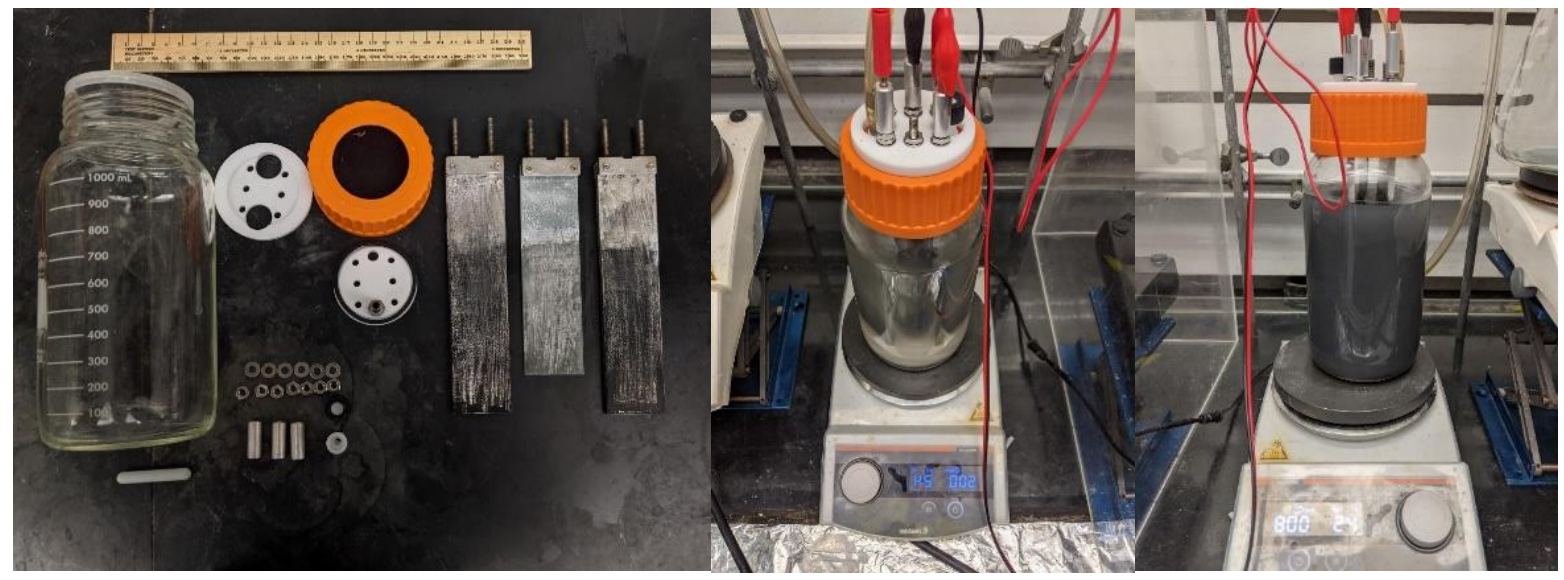

Figure S2. Left: Custom batch reactor disassembled. Middle: Assembled reactor top view. Right: Assembled reactor at reaction completion 


\section{Experimental Procedures}

\section{3-1. Table S1: Initial screening of viable poly(styrene) reduction conditions}<smiles>CC(C)C(C)CC(C)(C)C</smiles>

PS

[commercial grade, 174 kDA]
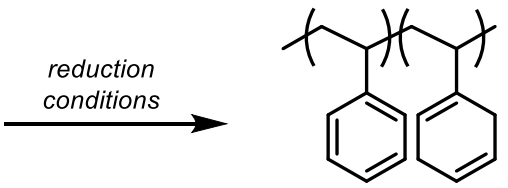

dearomatized PS

\begin{tabular}{|c|c|c|}
\hline entry & conditions & result \\
\hline 1 & $\begin{array}{l}\mathrm{Li}(1.0 \text { equiv. }) \\
\mathrm{NH}_{3} / \mathrm{THF} / t-\mathrm{BuOH},-78^{\circ} \mathrm{C} \\
\text { [Birch reduction] }\end{array}$ & no reaction \\
\hline 2 & $\begin{array}{l}\mathrm{Li}(5.0 \text { equiv.) } \\
\mathrm{NH}_{3} / \mathrm{THF} / t-\mathrm{BuOH},-33^{\circ} \mathrm{C} \\
\text { [Birch reduction] }\end{array}$ & no reaction \\
\hline 3 & $\begin{array}{c}\mathrm{Li}(5.0 \text { equiv. }) \\
\mathrm{Me}_{2} \mathrm{NH} /\left(\mathrm{CH}_{2} \mathrm{NH}_{2}\right)_{2}, 25^{\circ} \mathrm{C} \\
\text { [Benkeser reduction] }\end{array}$ & trace/complicated mixture \\
\hline 4 & $\begin{array}{c}\text { dimethylurea (3.0 equiv.) } \\
\text { TPPA ( } 8.0 \text { equiv.), GSW(-)/Mg(+) } \\
\text { electrons ( } 8.0 \text { equiv.), } 10 \mathrm{~mA} \\
\mathrm{LiBr} \text {, THF }(0.05 \mathrm{M}), 25^{\circ} \mathrm{C} \\
\text { [electroreduction - initial conditions] }\end{array}$ & $\begin{array}{l}21 \% \text { diene } \\
(25 \% \text { total })\end{array}$ \\
\hline
\end{tabular}

\section{3-2. Table S2: Optimized reaction conditions and selected optimization entries}
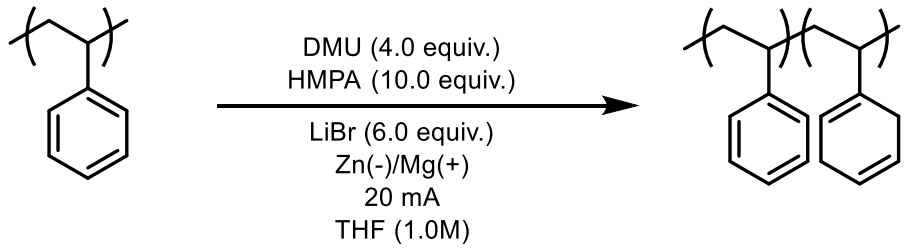

THF $(1.0 \mathrm{M})$

\begin{tabular}{|c|c|c|c|}
\hline Entry & $\begin{array}{c}\text { Deviation from } \\
\text { general conditions }\end{array}$ & $\mathrm{F} / \mathrm{mol}$ & Conversion $^{a}$ \\
\hline 1 & None & 1.0 & $20(24)$ \\
\hline 2 & $\mathrm{DCM}$ as solvent & 1.0 & $17(19)$ \\
\hline 3 & Ether as solvent & 1.0 & $14(17)$ \\
\hline 4 & $\mathrm{MeCN}$ as solvent & 1.0 & $20(24)$ \\
\hline 5 & 5.0 equiv. HMPA & 1.0 & $17(19)$ \\
\hline 6 & 1.0 equiv. HMPA & 1.0 & $14(17)$ \\
\hline 7 & 1.0 equiv. HMPA & 3.0 & decomposition \\
\hline 8 & No HMPA, 8.0 equiv. DMU & NA & N.R. - voltage limit exceeded \\
\hline 9 & 2.0 equiv. DMU & 1.0 & decomposition \\
\hline 10 & Mg (99.99\% purity) & 1.0 & $18(20)$ \\
\hline 11 & Open to Air & 1.0 & $11(13)$ \\
\hline 12 & reagents not dried & 1.0 & $12(15)$ \\
\hline 13 & [0.2 M] in polyarene, 1.0 equv. HMPA & 1.0 & $15(18)$ \\
\hline 14 & $60 \mathrm{~mA}$ & 1.0 & $19(22)$ \\
\hline 15 & 12 equiv. $\mathrm{LiBr}$ & NA & N.R - lithium metal build up on anode \\
\hline 16 & $-78^{\circ} \mathrm{C}$ & 4.0 & $70(74)$ \\
\hline
\end{tabular}

${ }^{a}$ Conversion to diene determined by ${ }^{1} \mathrm{H}$ NMR. Total reduction, conversion to diene and overreduced products shown in parentheses. Entry 1 was chosen as general optimized conditions for any anticipated degree of conversion. Entry 10 was chosen as optimized conditions for $1.0 \mathrm{~F} / \mathrm{mol}$ due to increased concentration and decreased HMPA loadings. 


\section{3-3. General procedure for the dearomatization of polyarenes}



This procedure was modified from the literature protocol. ${ }^{2}$

The magnesium anode was pre-inserted into the septa of a $40 \mathrm{~mL}$ oven-dried vial cap and transferred into the glovebox. Inside a nitrogen-filled glovebox, a $40 \mathrm{~mL}$ vial was charged with polymer $(1.9 \mathrm{mmol}$ of arene units, 1.0 equiv.), dimethylurea (677 mg, $7.7 \mathrm{mmol}, 4.0$ equiv.), and lithium bromide (1.0 g, $11.5 \mathrm{mmol}, 6.0$ equiv.). The vial cap was screwed on to the reaction vessel, sealed with electrical tape, and brought out of the glovebox. Tetrahydrofuran $(19.2 \mathrm{~mL})$ and hexamethylphosphoramide $(3.4 \mathrm{~g}, 3.3 \mathrm{~mL}, 19.2 \mathrm{mmol}, 10$ equiv.) were added, and the reaction was stirred until complete dissolution of reagents (approximately 515 minutes). During this time, the zinc cathode was inserted through the septa, parallel to the magnesium anode, and 20 milliamps of current was applied, using a DC power source. The reaction was run with high stirring $(1000 \mathrm{rpm})$ at room temperature for the allotted reaction time.

Workup A: The DC power supply was turned off and the reaction mixture was poured into a stirring solution of $\mathrm{HCl}$ in methanol $(60 \mathrm{~mL}, 0.05 \mathrm{M}$, prepared from aqueous $37 \% \mathrm{HCl})$. The reaction vessel and electrodes were washed with DCM (approximately $3 \mathrm{~mL}$ ), which was then added to the beaker containing methanol and crude product. This mixture was stirred for 1 minute, filtered using a sintered filter funnel, and dried under a blanket of nitrogen for 20 minutes. The filter cake containing crude product was redissolved in DCM $(5 \mathrm{~mL})$, stirred until complete dissolution (approximately 5 minutes), poured into a stirring solution of methanol $(30 \mathrm{~mL})$, and the precipitate was filtered using a sintered filter funnel under a flow of nitrogen for 20 minutes. The filter cake (now pure product) was collected and dried under vacuum at room temperature for three hours before analysis.

Work-up B: The DC power supply was turned off and the reaction mixture was partitioned between diethyl ether $(10 \mathrm{~mL})$ and $\mathrm{HCl}$ (aq., $10 \mathrm{~mL}, 0.05 \mathrm{M})$ and the aqueous layer was extracted with diethyl ether $(3 \times 10$ $\mathrm{mL})$. The combined organic layers were washed with brine $(10 \mathrm{~mL})$, dried over $\mathrm{MgSO}_{4}$, and evaporated to approximately $3 \mathrm{~mL}$. The residue was poured into $\mathrm{H}_{2} \mathrm{O}(20 \mathrm{~mL})$ to precipitate the product and filtered under a flow of nitrogen for 20 minutes. The product was collected and dried under vacuum at room temperature for three hours before analysis.

Note: All products were stored under nitrogen in a $-20{ }^{\circ} \mathrm{C}$ freezer.

\section{Structure determination:}

Verification of the cyclohexadiene units was performed by 2D-NMR. The peaks at 5.7 and $5.3 \mathrm{ppm}$ were assigned as the secondary and tertiary olefin C-H's, respectively by HSQC. The lack of HMBC signals from the secondary proton $\mathrm{C}-\mathrm{H}$ 's to the tertiary $\mathrm{C}-\mathrm{H}$ allylic verified the 1,4-relationship between olefins. Assignment of the allylic protons was ambiguous. Each allylic $\mathrm{C}-\mathrm{H}$ peak in the ${ }^{1} \mathrm{H}$ NMR correlates to one carbon, as determined by HSQC, but no cross-peaks to olefins were observed. A HMBC cross-peak between both sets of allylic $\mathrm{C}-\mathrm{H}$ 's and the tertiary $\mathrm{C}=\mathbf{C}(\mathrm{CH}) \mathrm{CH}_{2}$ was observed; however, an HMBC cross peak from the one set of allylic C-H's to the allylic carbon on the backbone that would be expected was not observed. 
Determination of conversion and overreduction:

Conversion to the diene was determined by normalizing the peak area at $5.7 \mathrm{ppm}$, corresponding to the two secondary olefin C-H's, to 2.0. The arene region from 7.25-6.20 ppm was equivalent to 5 protons. Overreduction was calculated by subtracting the peaks associated with the arene monomer unit, diene monomer unit, and residual water from the alkyl region, and dividing the resulting number by 11 - an estimated number of protons contained in the region of $2.2 \mathrm{ppm}-1.0 \mathrm{ppm}$ for the overreduced product.

[The accuracy of this approach was also validated by comparison to ${ }^{1} \mathrm{H} N \mathrm{NM}$ utilizing $\mathrm{MeNO}_{2}$ as an internal standard (32 scans, $d 1=10$ seconds), which was in excellent agreement with the above method, and accounted for the mass balance.]

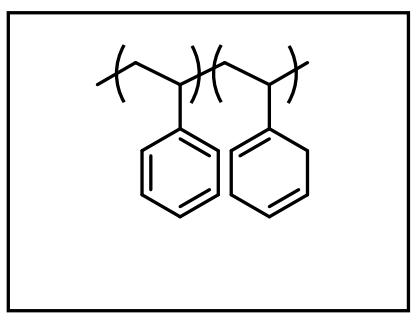

Monodisperse poly(styrene):

Monodisperse poly(styrene) ( $\mathrm{Mw}=16.7 \mathrm{kDA}, \mathrm{PDI}=1.06)(200 \mathrm{mg}, 1.92 \mathrm{mmol}$ arene, 1 equiv.) was subjected to standard conditions following work-up A. The results are tabulated below:

Equivalents

Conversion Overall

of electrons Time $(\mathrm{h})$ to diene $(\%)$ conversion (\%)

$\begin{array}{ccc}\mathrm{Tg}\left({ }^{\circ} \mathrm{C}\right) & \mathrm{Mw}(\mathrm{kDa}) & \mathrm{PDI} \\ 101 & 16.7 & 1.06 \\ 98 & 16.5 & 1.06 \\ 96 & 16.7 & 1.07 \\ 95 & 16.8 & 1.08 \\ 95 & 16.3 & 1.07\end{array}$



Commercial poly(styrene):

Commercial poly(styrene) $(\mathrm{Mw}=174 \mathrm{kDa}, \mathrm{PDI}=2.61)(200 \mathrm{mg}, 1.92 \mathrm{mmol}$ arene, 1 equiv.) was subjected to standard conditions following work-up A.

The results are tabulated below:

\begin{tabular}{ccccccc}
$\begin{array}{c}\text { Equivalents } \\
\text { of electrons }\end{array}$ & Time $(\mathrm{h})$ & $\begin{array}{c}\text { Conversion } \\
\text { to diene }(\%)\end{array}$ & $\begin{array}{c}\text { Overall } \\
\text { conversion }(\%)\end{array}$ & $\mathrm{Tg}\left({ }^{\circ} \mathrm{C}\right)$ & $\mathrm{Mw}(\mathrm{kDa})$ & $\mathrm{PDI}$ \\
\hline 0 & - & - & - & 103 & 174 & 2.61 \\
1 & 2.57 & 20 & 24 & 103 & 179 & 2.58 \\
2 & 5.14 & 32 & 38 & 103 & 172 & 2.66 \\
3 & 7.72 & 44 & 56 & 101 & 163 & 2.61 \\
4 & 10.29 & 57 & 69 & 100 & 147 & 2.62
\end{tabular}


For partial conversion:

${ }^{1} \mathrm{H}$ NMR: $\left(500 \mathrm{MHz}, \mathrm{CDCl}_{3}\right) \delta 7.11,7.07,6.96,6.69,6.59,6.52,6.47,5.67,5.27,2.64,2.44,1.93,1.86$, $1.76,1.52,1.43,1.20$.

${ }^{13} \mathrm{C}$ NMR: $\left(126 \mathrm{MHz}, \mathrm{CDCl}_{3}\right) \delta 145.8,137.5,128.1,128.0,127.8,127.6,127.4,125.8,125.6,124.6,124.4$, $120.4,46.2,44.3,44.0,42.2,40.6,37.9,26.9,25.6,25.1,23.3$.

IR: (ATR, neat, $\left.\mathrm{cm}^{-1}\right)$ 3059, 3025, 2922, 2819, 2852, 1601, 1493, 1452, 1362, 957, 758

For complete conversion:

${ }^{1} \mathrm{H}$ NMR: $\left(500 \mathrm{MHz}, \mathrm{CDCl}_{3}\right) \delta 7.20,7.12,7.02,5.68,5.28,2.64,2.45,1.95,1.87,1.76,1.54,1.20$

${ }^{13} \mathrm{C}$ NMR: $\left(126 \mathrm{MHz}, \mathrm{CDCl}_{3}\right) \delta 137.4,136.2,128.0,124.6,124.4,123.2,120.3,119.5,42.3,37.9,26.9$, 25.6, 25.2, 24.0, 23.3.

IR: (ATR, neat, $\left.\mathrm{cm}^{-1}\right)$ 3025, 2922, 2882, 2852, 2819, 1597, 1493, 1452, 1428, 1028, 957, 907, 758
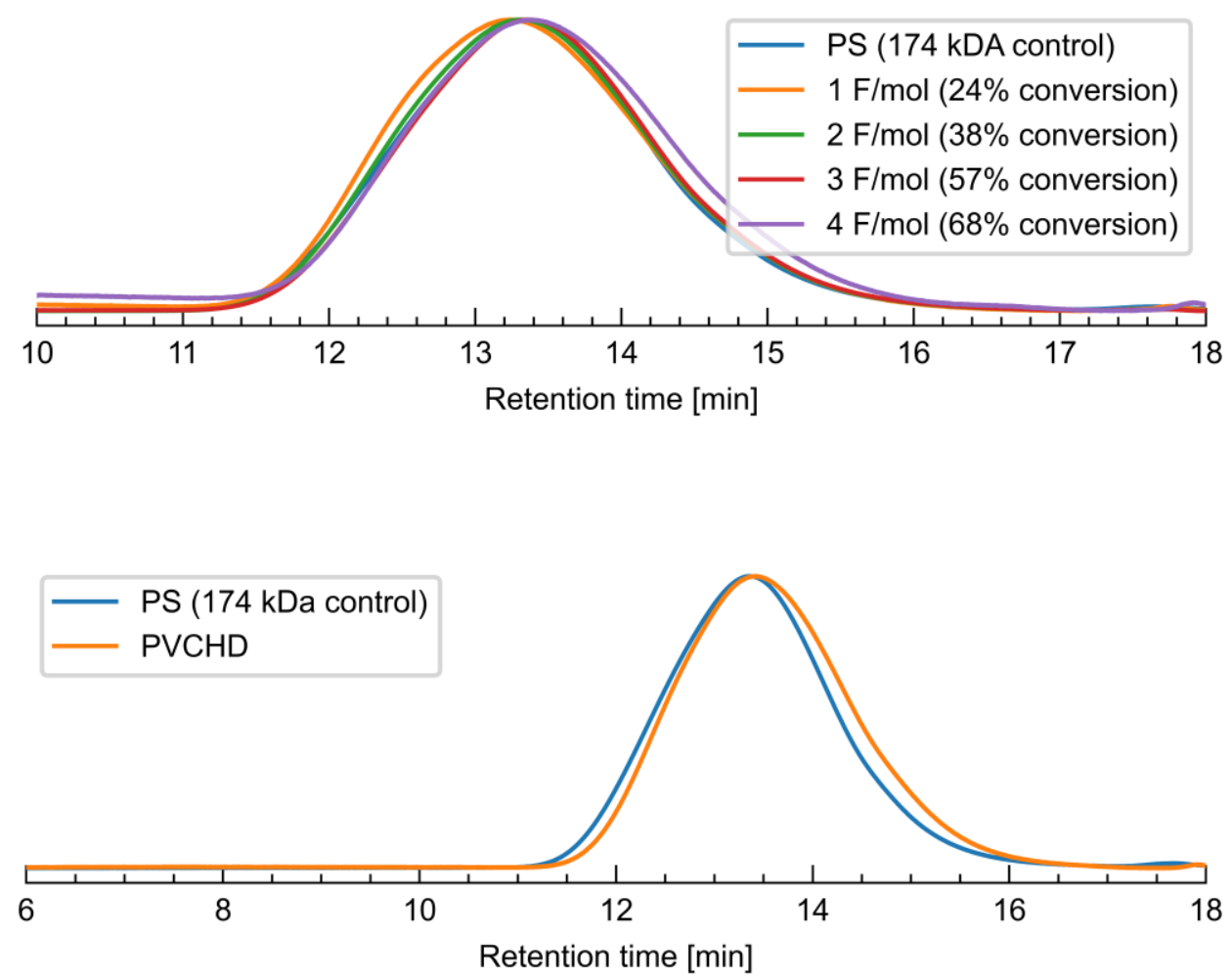

Figure S3. Top: GPC traces of partially converted commercial poly(styrene) with varying F/mol. Bottom: GPC trace of poly(vinylcyclohexadiene) overlayed with parent polymer. 


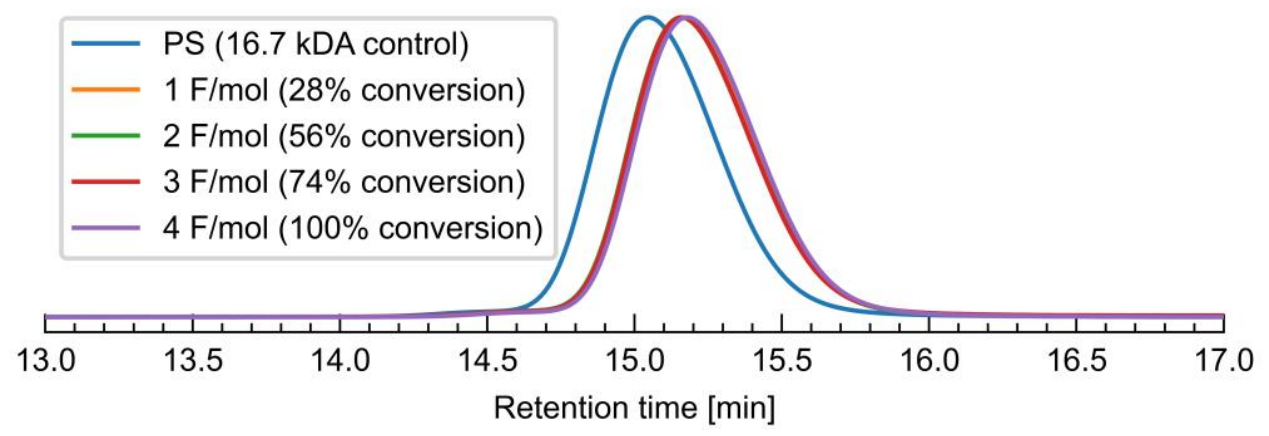

Figure S4. GPC traces of partially converted monodisperse low Mw poly(styrene) with varying F/mol. 


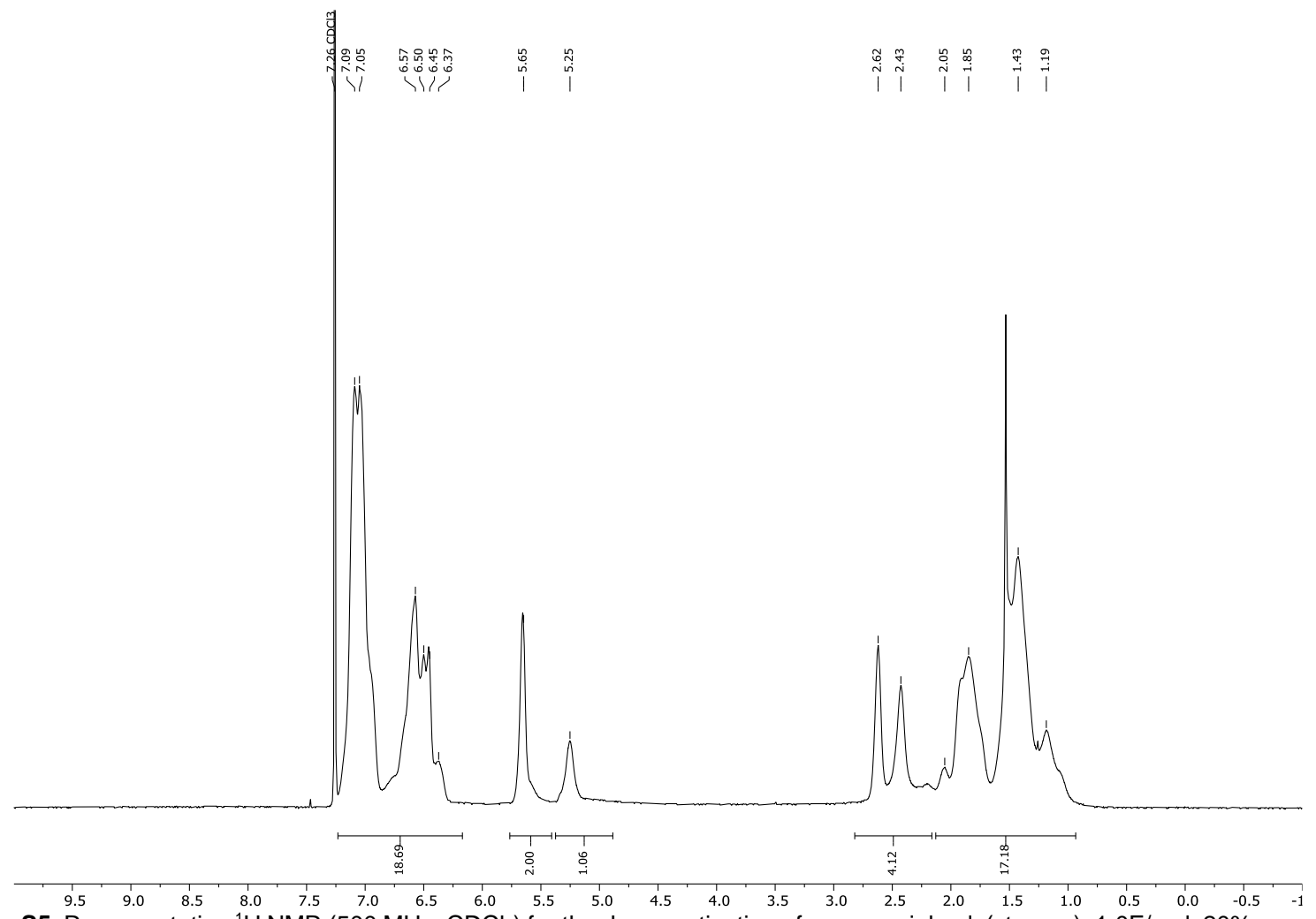

Figure S5. Representative ${ }^{1} \mathrm{H}$ NMR $\left(500 \mathrm{MHz}, \mathrm{CDCl}_{3}\right.$ ) for the dearomatization of commercial poly(styrene): $1.0 \mathrm{~F} / \mathrm{mol}, 20 \%$ conversion to diene, $24 \%$ conversion to diene and overreduced product.
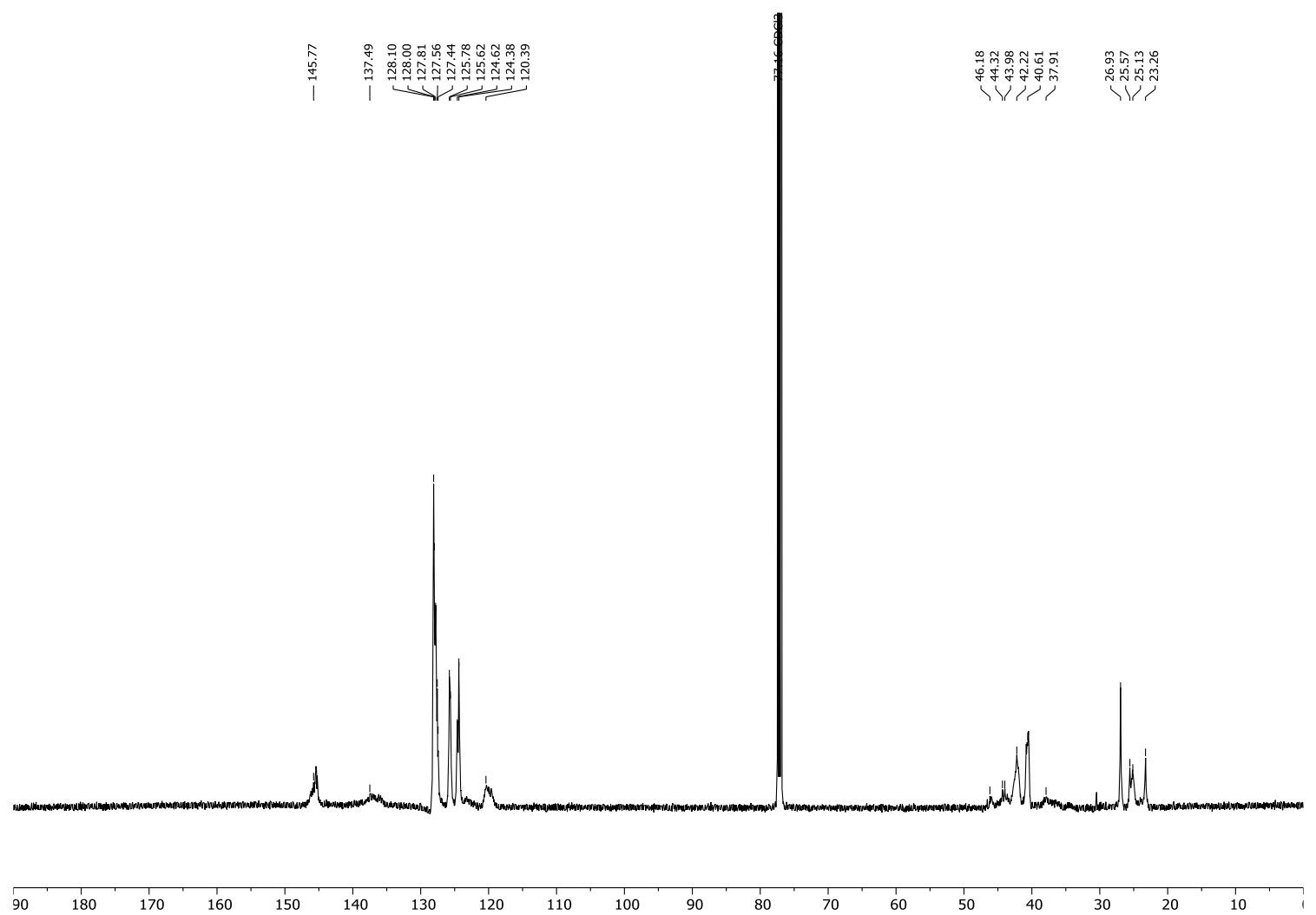

Figure S6. Representative ${ }^{13} \mathrm{C} \mathrm{NMR}\left(126 \mathrm{MHz}_{2} \mathrm{CDCl}_{3}\right)$ for the dearomatization of commercial poly(styrene): $1.0 \mathrm{~F} / \mathrm{mol}, 20 \%$ conversion to diene, $24 \%$ conversion to diene and overreduced product. 


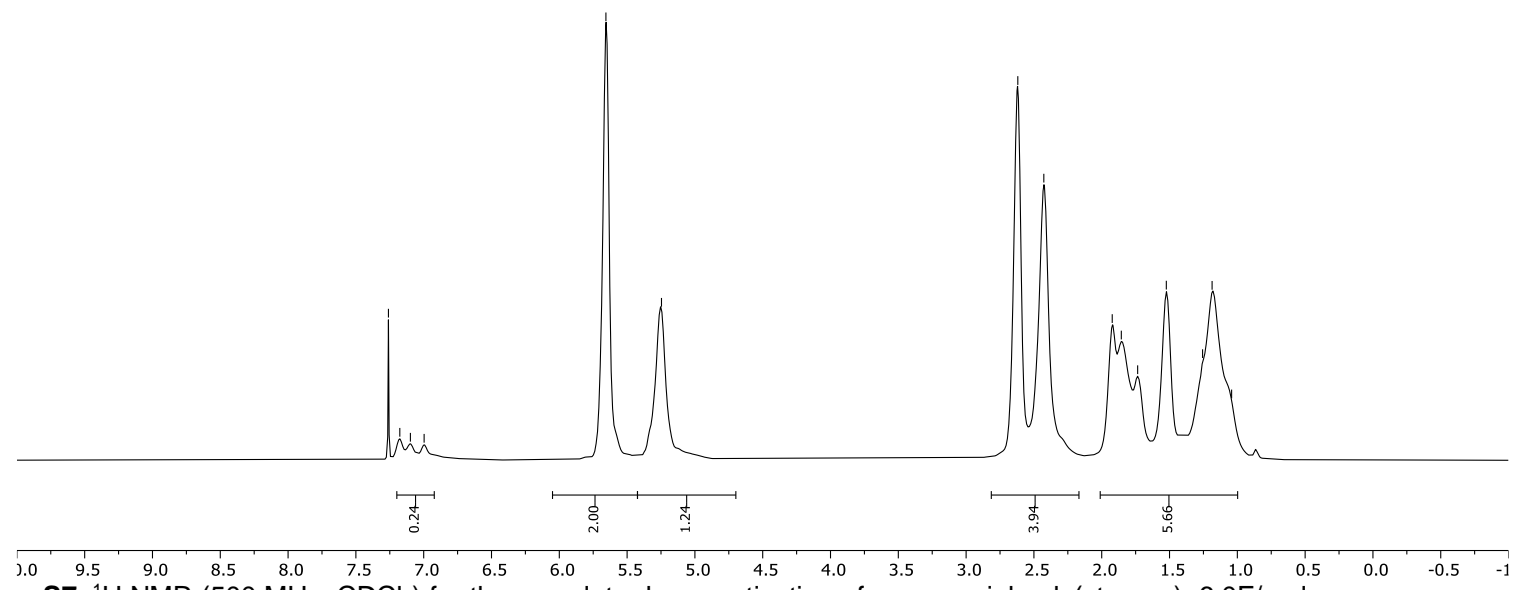

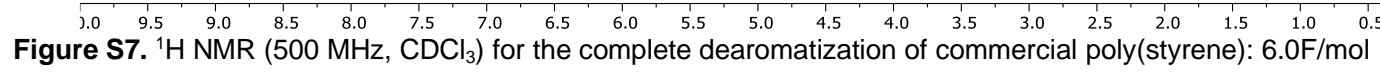

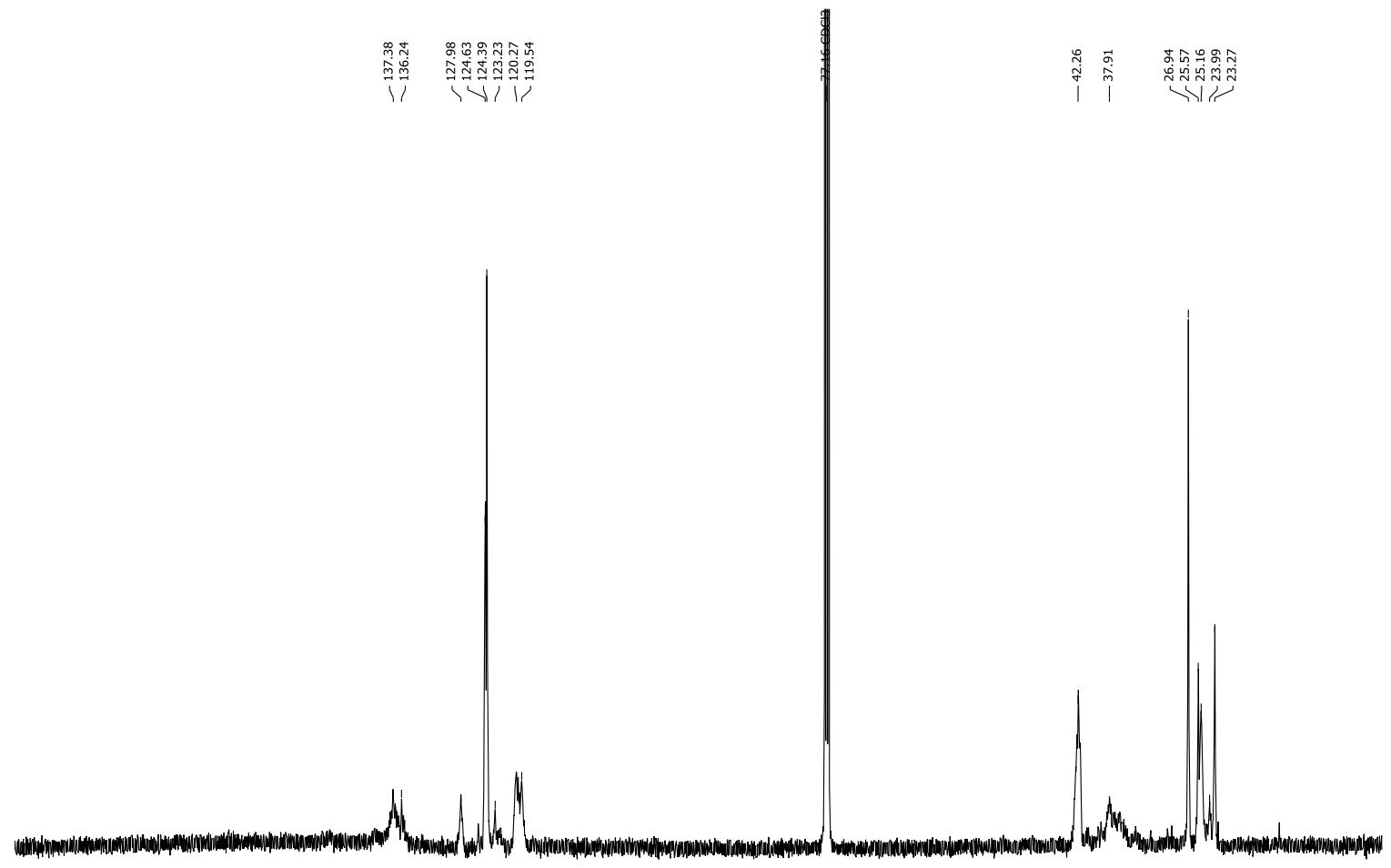

Figure S8. ${ }^{13} \mathrm{C}$ NMR $\left(500 \mathrm{MHz}, \mathrm{CDCl}_{3}\right.$ ) 


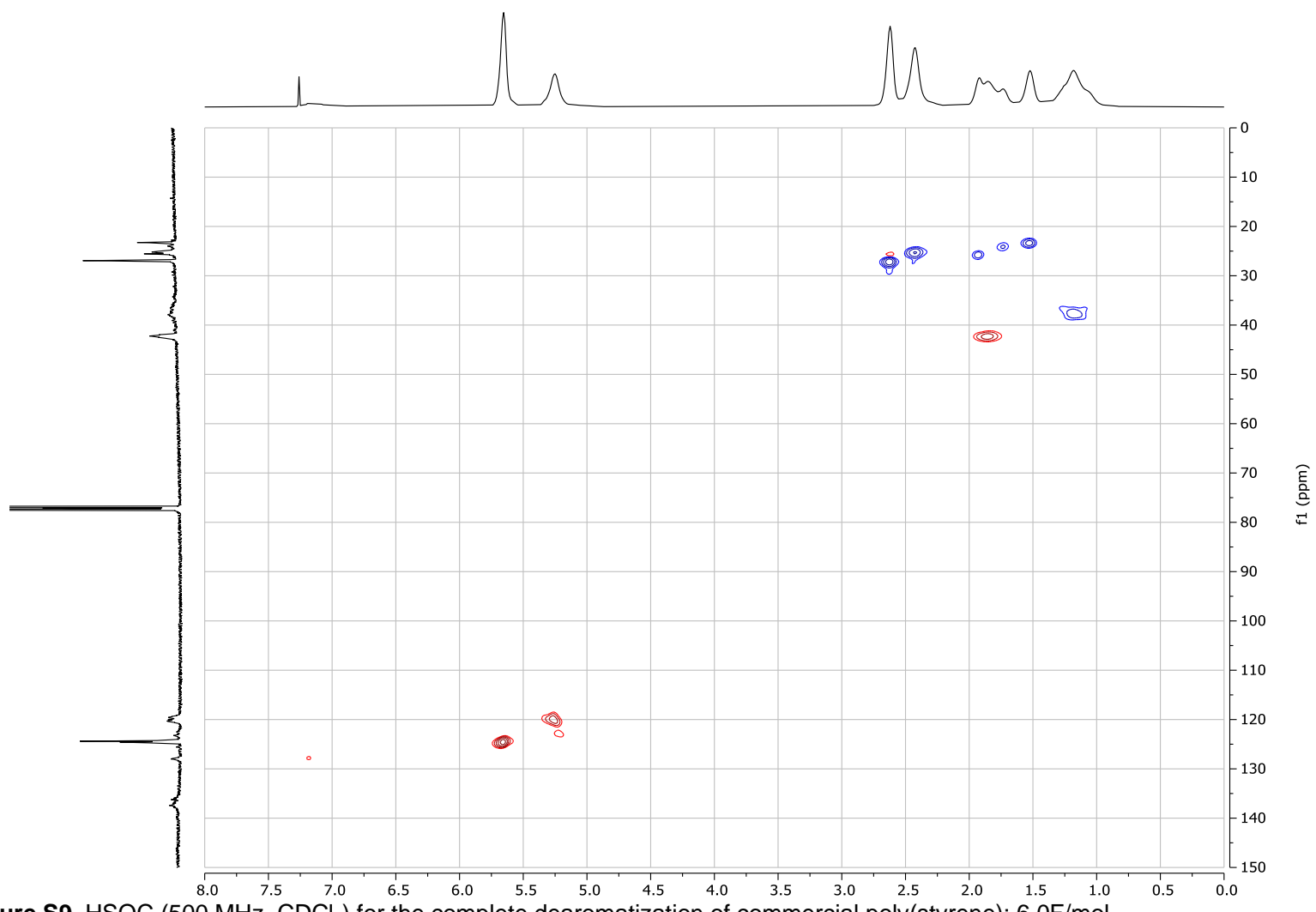

Figure S9. HSQC $\left(500 \mathrm{MHz}, \mathrm{CDCl}_{3}\right)$ for the complete dearomatization of commercial poly(styrene): $6.0 \mathrm{~F} / \mathrm{mol}$



Figure S10. $\mathrm{HMBC}\left(500 \mathrm{MHz}, \mathrm{CDCl}_{3}\right)$ for the complete dearomatization of commercial poly(styrene): $6.0 \mathrm{~F} / \mathrm{mol}$ 


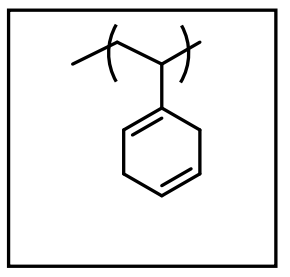

Commercial poly(styrene) - complete conversion:

The reaction was modified from the general procedure as follows:

Poly(styrene) (100 mg, 1.0 equiv.), dimethylurea (339 mg, 4.0 equiv.), $\mathrm{LiBr}(500 \mathrm{mg}$, 6.0 equiv.), HMPA (1.7g, 10 equiv.), THF (19.2 mL). After $6.0 \mathrm{~F} / \mathrm{mol}(7,68 \mathrm{~h}), 79 \%$ conversion to the diene (95\% to diene and overreduced products, $100 \%$ overall conversion) was observed.

${ }^{1} \mathrm{H}$ NMR: $\left(500 \mathrm{MHz}, \mathrm{CDCl}_{3}\right) \delta 7.20,7.12,7.02,5.68,5.28,2.64,2.45,1.95,1.87,1.76,1.54,1.20$

${ }^{13} \mathrm{C}$ NMR: $\left(126 \mathrm{MHz}, \mathrm{CDCl}_{3}\right) \delta 137.4,136.2,128.0,124.6,124.4,123.2,120.3,119.5,42.3,37.9,26.9$, 25.6, 25.2, 24.0, 23.3.

DSC: $\left({ }^{\circ} \mathrm{C}, 10^{\circ} \mathrm{C} / \mathrm{min}\right): 97^{\circ} \mathrm{C}$

GPC: $(\mathrm{THF}) \mathrm{Mw}=158 \mathrm{kDa}, \mathrm{PDI}=2.60$

TGA: $\left({ }^{\circ} \mathrm{C}, 15^{\circ} \mathrm{C} / \mathrm{min}\right) \mathrm{T}_{\text {deg }}=380^{\circ} \mathrm{C}$

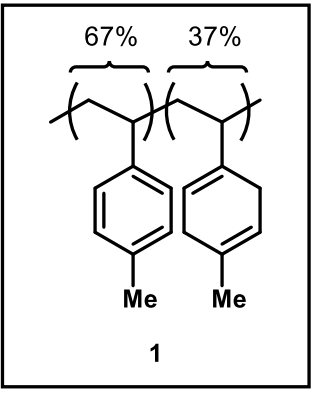

1: Poly(4-methylstyrene) ( $\mathrm{Mw}=73.0 \mathrm{kDa}, \mathrm{PDI}=3.30)(227 \mathrm{mg}, 1.92 \mathrm{mmol}$ of arene repeat unit) was subjected to standard conditions following work-up A. After 2.0 $\mathrm{F} / \mathrm{mol}(5.12 \mathrm{~h}), 37 \%$ conversion to the diene (43\% overall) was observed.

${ }^{1} \mathrm{H}$ NMR: $\left(500 \mathrm{MHz}, \mathrm{CDCl}_{3}\right) \delta 6.91,6.84,6.75,6.52,6.47,6.40,5.38,5.25,2.52,2.42,2.30,2.24,1.86$, $1.68,1.39,1.16,0.94$.

${ }^{13} \mathrm{C}$ NMR: $\left(126 \mathrm{MHz}, \mathrm{CDCl}_{3}\right) \delta 142.7,134.8,134.6,131.1,129.0,128.8,128.7,127.7,127.4,119.0,41.8$, 40.0, 31.8, 26.07, 23.2, 22.0, 21.2, 21.1.

GPC: $(\mathrm{THF}) \mathrm{Mw}=75.3 \mathrm{kDa}, \mathrm{PDI}=3.27$

IR: (ATR, neat, $\left.\mathrm{cm}^{-1}\right)$ 3017, 2919, 2851, 2814, 1512, 1447, 1375, 1112, 1037, 1020, 947, 813, 781, 723

DSC: $\left({ }^{\circ} \mathrm{C}, 10^{\circ} \mathrm{C} / \mathrm{min}\right)$ Parent: $T_{g 1}=102^{\circ} \mathrm{C} T_{g 2}=113^{\circ} \mathrm{C}$, Functionalized: $T_{g}=102^{\circ} \mathrm{C}, 114{ }^{\circ} \mathrm{C}$

TGA: $\left({ }^{\circ} \mathrm{C}, 15^{\circ} \mathrm{C} / \mathrm{min}\right) \mathrm{T}_{\text {deg }}=386^{\circ} \mathrm{C}$ 


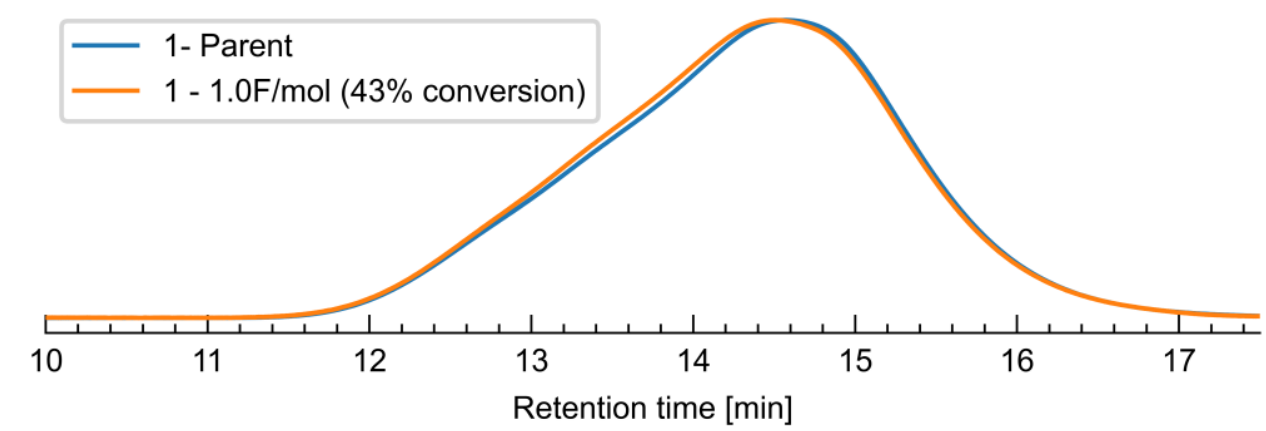

Figure S11. GPC trace of 1 overlayed with parent polymer. 


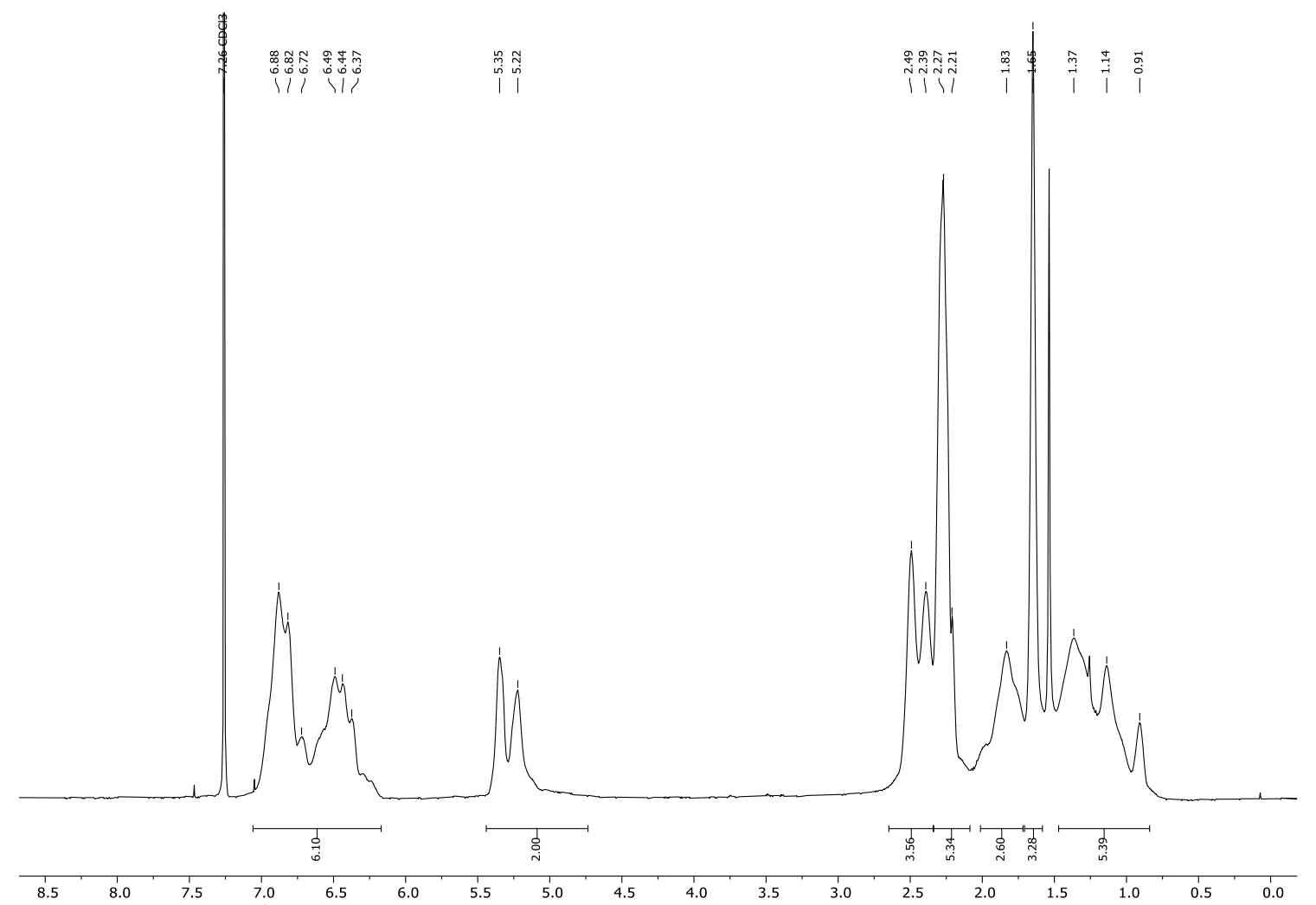

Figure S12. Representative ${ }^{1} \mathrm{H}-\mathrm{NMR}\left(500 \mathrm{MHz}, \mathrm{CDCl}_{3}\right)$ for the dearomatization of Poly(4-methylstyrene) to 1:2.0 F/mol



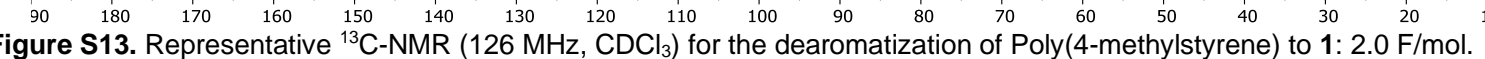




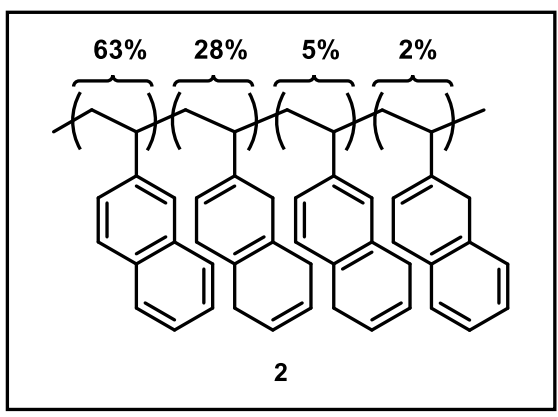

2: Poly(2-vinyl-naphthalene) $(\mathrm{Mw}=51 \mathrm{kDa}, \mathrm{PDI}=1.82)(296 \mathrm{mg}$, $1.92 \mathrm{mmol}$ of arene repeat unit) was subjected to standard conditions following work-up A. After $4.0 \mathrm{~F} / \mathrm{mol}(10.29 \mathrm{~h}), 36 \%$ conversion to a 1:0.2:0.08 ratio of the bis-reduced: secondary olefin: tertiary olefin products was observed.

${ }^{1}$ H NMR: $\left(500 \mathrm{MHz}, \mathrm{CDCl}_{3}\right) \delta 7.60,7.31,7.10,6.97,6.67,6.55,6.45,5.87,5.71,5.45,5.22,3.29,2.66$, $2.49,2.25,2.00,1.85,1.62,1.48,1.34,1.20,1.05$.

${ }^{13} \mathrm{C}$ NMR: $\left(126 \mathrm{MHz}, \mathrm{CDCl}_{3}\right) \delta 142.3,133.3,132.1,127.6,127.4,126.7,125.6,125.0,124.7,123.3,42.0$, 40.7, 32.0, 31.2, 30.8, 29.9, 23.5.

GPC (THF) Mw $=48.8 \mathrm{kDa}, \mathrm{PDI}=1.98$

IR: (ATR, neat, $\left.\mathrm{cm}^{-1}\right)$ 3053, 3034, 2919, 1638, 1599, 1507, 1125, 1018, 969, 947, 855

DSC: $\left({ }^{\circ} \mathrm{C}, 10^{\circ} \mathrm{C} / \mathrm{min}\right)$ Parent: $T_{g}=121^{\circ} \mathrm{C}$, Functionalized: $T_{g}=123^{\circ} \mathrm{C}$

TGA: $\left({ }^{\circ} \mathrm{C}, 15^{\circ} \mathrm{C} / \mathrm{min}\right) \mathrm{T}_{\text {deg }}=406^{\circ} \mathrm{C}$

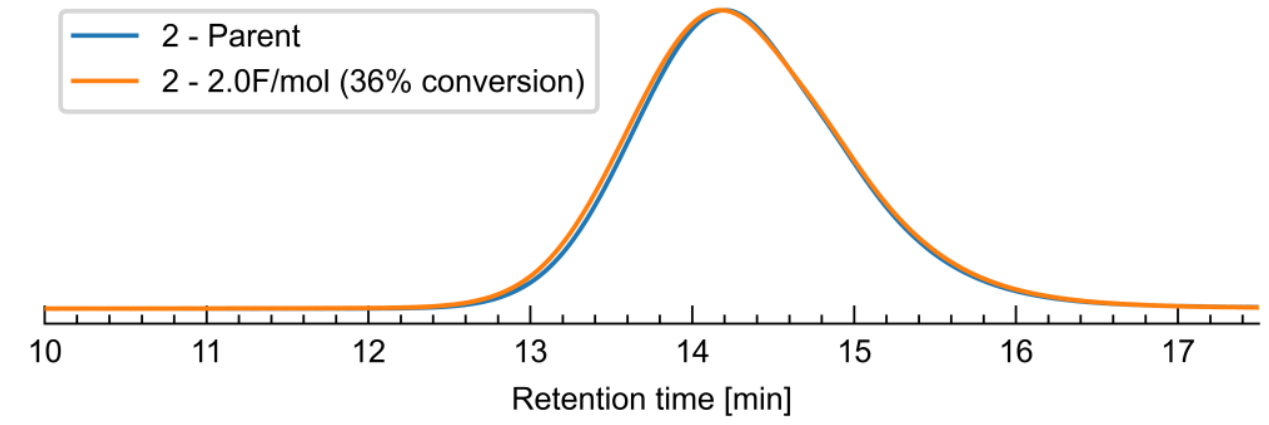

Figure S14. GPC trace of 2 overlayed with parent polymer. 



Figure S15. Representative ${ }^{1} \mathrm{H}-\mathrm{NMR}\left(500 \mathrm{MHz}, \mathrm{CDCl}_{3}\right)$ for the dearomatization of Poly(2-vinyl-naphthalene) to 2: $2.0 \mathrm{~F} / \mathrm{mol}$.
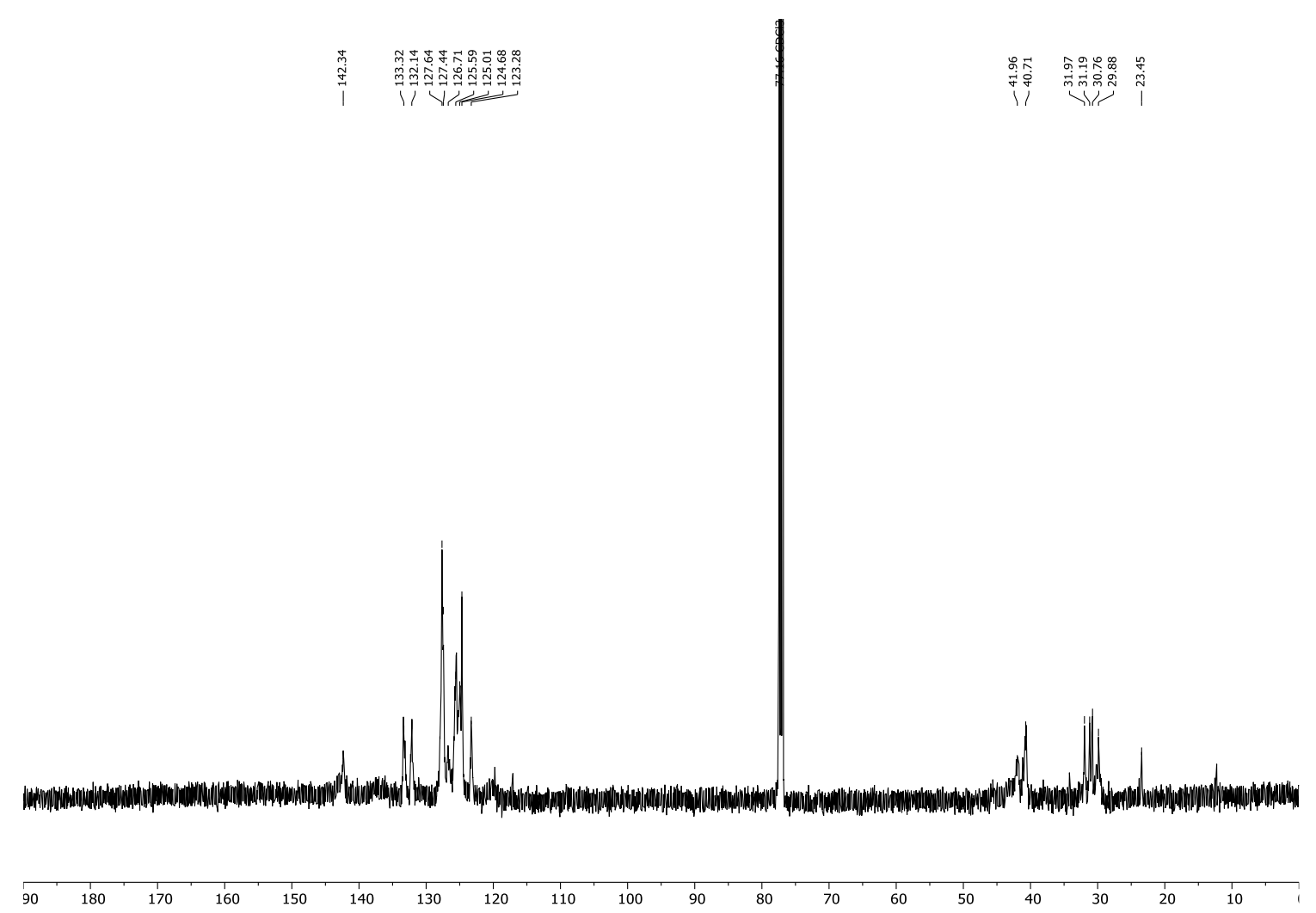

Figure S16. Representative ${ }^{13} \mathrm{C}-\mathrm{NMR}\left(126 \mathrm{MHz}, \mathrm{CDCl}_{3}\right)$ for the dearomatization of Poly(2-vinyl-naphthalene) to 2: $2.0 \mathrm{~F} / \mathrm{mol}$. 


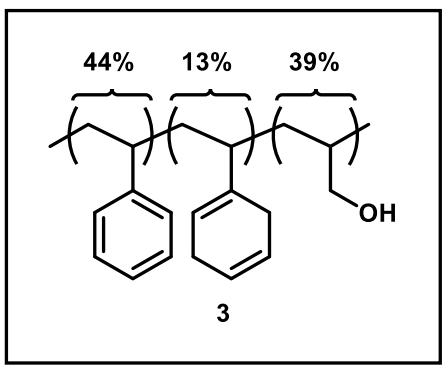

3: Poly(styrene-co-allyl-alcohol), possessing a 1:0.65 molar ratio of styrene: allyl alcohol units $(\mathrm{Mw}=2.77 \mathrm{kDa}, \mathrm{PDI}=1.875)(272 \mathrm{mg}, 1.92 \mathrm{mmol}$ of arene repeat unit) was subjected to standard conditions following work-up A. After $1.0 \mathrm{~F} / \mathrm{mol}(2.56 \mathrm{~h}), 22 \%$ conversion to the diene (27\% overall) was observed. Residual HMPA was present after 3 precipitations from water and attempted chromatographic separation.

'H NMR: $\left(500 \mathrm{MHz}, \mathrm{CDCl}_{3}\right) \delta$ 7.14, 6.82, 6.68, 5.67, 5.37, 5.27, 3.58, 3.44, 2.45, 1.95, 1.75, 1.54.

${ }^{13} \mathrm{C}$ NMR: $\left(126 \mathrm{MHz}, \mathrm{CDCl}_{3}\right) \delta 145.3,128.3,127.8,126.1,124.4,68.1,40.8,29.9,27.5,26.9,25.7,23.2$.

IR: (ATR, neat, $\left.\mathrm{cm}^{-1}\right)$ 3328, 2920, 2851, 1659, 1605, 1493, 1452, 1298, 1197, 1029, 985, 957, 918

GPC (THF) $\mathrm{Mw}=2.52 \mathrm{kDa}, \mathrm{PDI}=1.796$

DSC: $\left({ }^{\circ} \mathrm{C}, 10{ }^{\circ} \mathrm{C} / \mathrm{min}\right)$ Parent $: T_{g}=63{ }^{\circ} \mathrm{C}$, Functionalized : $T_{g}=42{ }^{\circ} \mathrm{C}-$ inconclusive due to polymer contamination

TGA: $\left({ }^{\circ} \mathrm{C}, 15^{\circ} \mathrm{C} / \mathrm{min}\right) \mathrm{T}_{\text {solvent }}=173^{\circ} \mathrm{C}, \mathrm{T}_{\text {deg }}=210^{\circ} \mathrm{C}$



Figure S17. GPC trace of $\mathbf{3}$ overlayed with parent polymer. 


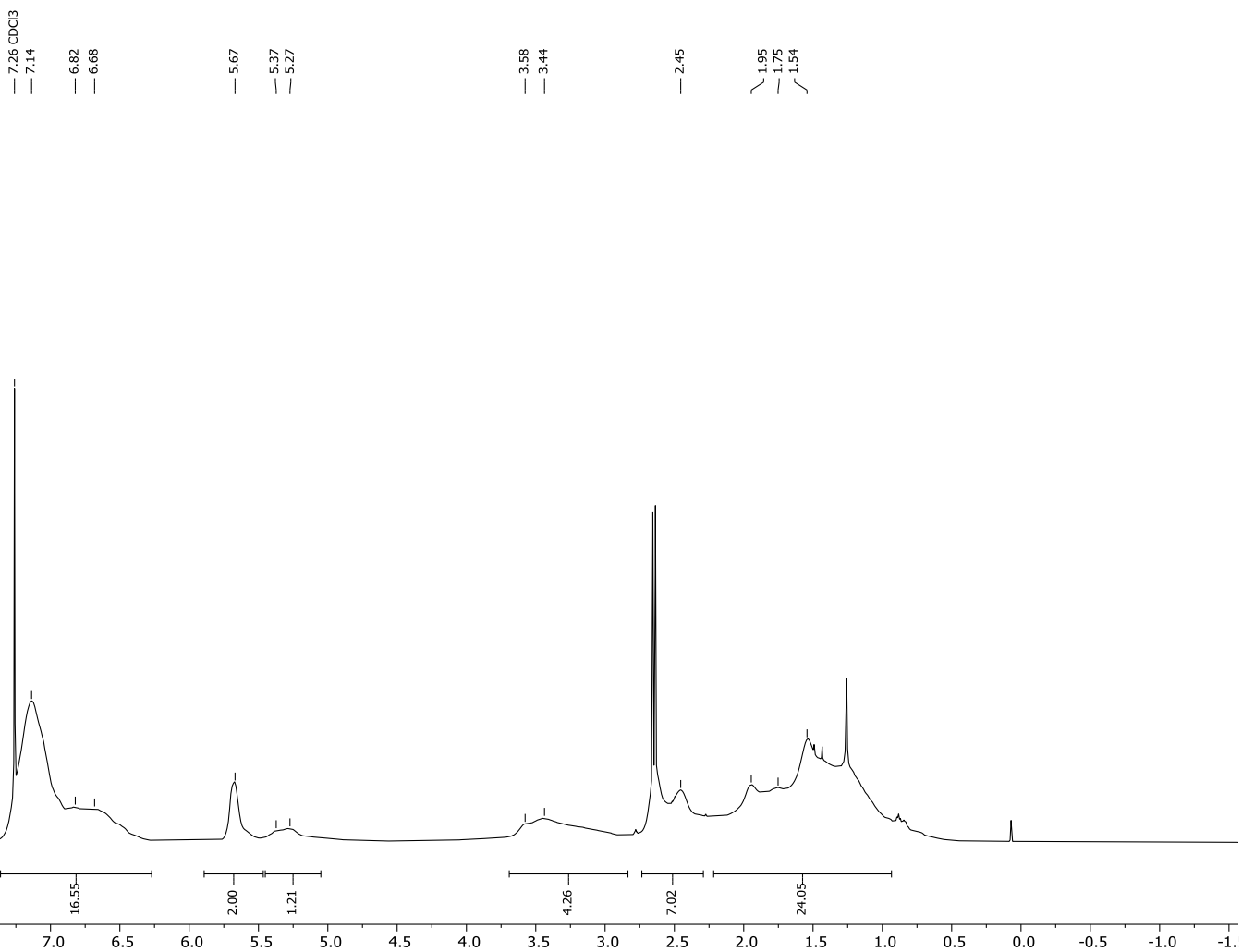

Figure S18. Representative ${ }^{1} \mathrm{H}-\mathrm{NMR}\left(500 \mathrm{MHz}, \mathrm{CDCl}_{3}\right)$ for the dearomatization of Poly(styrene-co-allyl-alcohol) to 3: $1.0 \mathrm{~F} / \mathrm{mol}$.

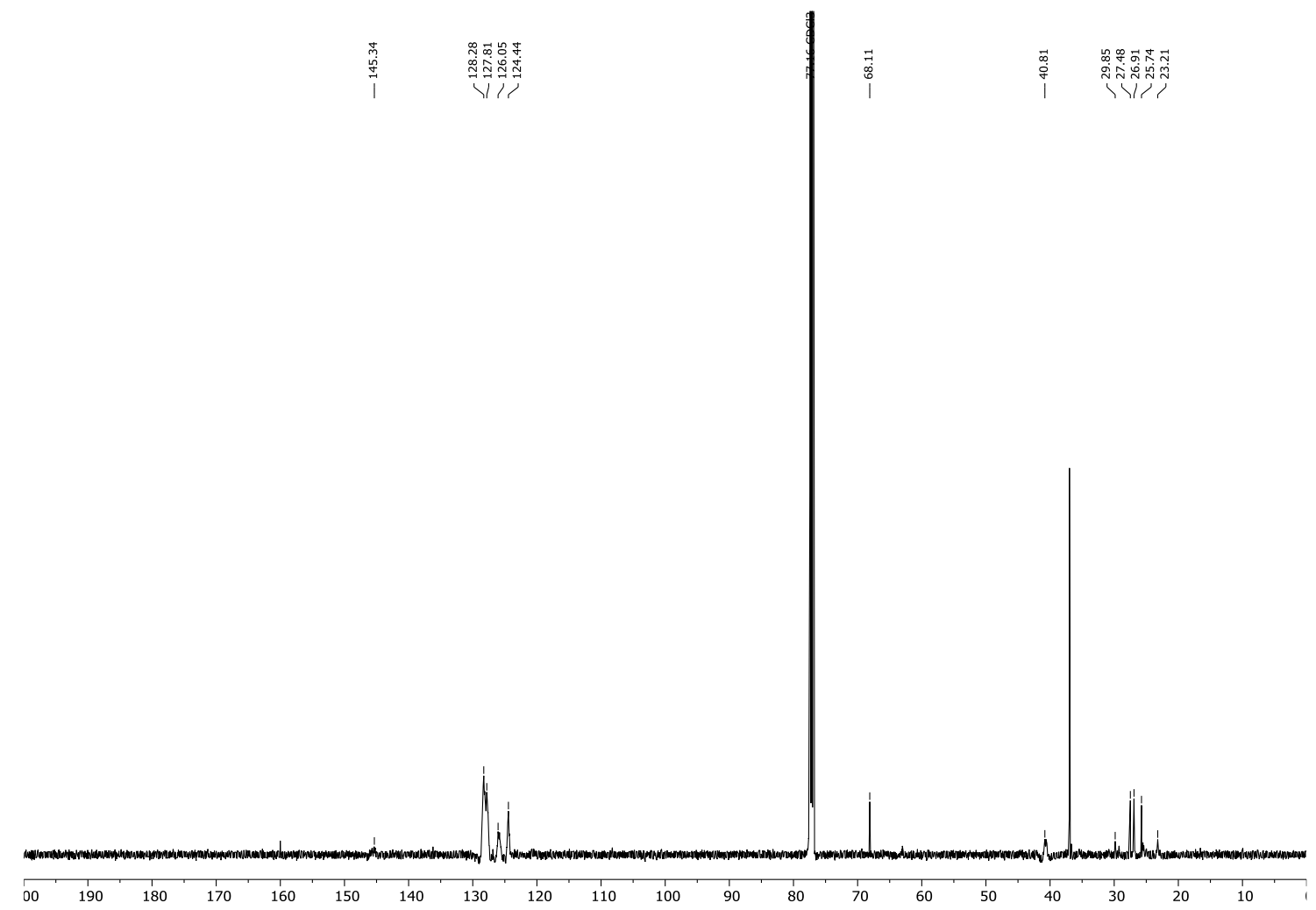

Figure S19. Representative ${ }^{13} \mathrm{C}-\mathrm{NMR}\left(126 \mathrm{MHz}, \mathrm{CDCl}_{3}\right)$ for the dearomatization of Poly(styrene-co-allyl-alcohol) to 3: $1.0 \mathrm{~F} / \mathrm{mol}$. 


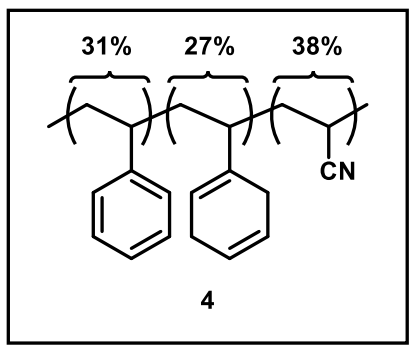

4: Poly(styrene-co-acrylonitrile) $(\mathrm{Mw}=142 \mathrm{kDa}, \mathrm{PDI}=2.87)$, possessing a 1.0:0.6 molar ratio of styrene: acrylonitrile units, $(261 \mathrm{mg}, 1.92 \mathrm{mmol}$ of arene repeat unit) was subjected to standard conditions following work-up B. After 2.0 F/mol (5.12 h), $43 \%$ conversion to the diene (51\% overall) was observed. A noticeable change in the MWD was observed in this case, and while nitrile decomposition cannot be ruled out, there is evidence for the retention of the nitrile moiety. A distinctive nitrile $\mathrm{C} \equiv \mathrm{N}$ stretch was observed at $2229 \mathrm{~cm}^{-1}$, as well as a nitrile $\mathrm{CHCN}$ peak at $118.1,118.4 \mathrm{ppm}$ in the ${ }^{13} \mathrm{C}-\mathrm{NMR}$. No distinctive amine $\mathrm{N}-\mathrm{H}$ stretches were observed in the IR spectra, tentatively ruling out overreduction of the nitrile.

${ }^{1} \mathrm{H}$ NMR: $\left(500 \mathrm{MHz}, \mathrm{CDCl}_{3}\right) \delta$ 7.14, 6.75, 5.70, 5.38, 2.67, 2.58, 2.48, 1.78, 1.42, 1.40, 1.26, 0.85.

${ }^{13} \mathrm{C}$ NMR: $\left(126 \mathrm{MHz}, \mathrm{CDCl}_{3}\right) \delta 128.9,128.5,128.3,127.7,127.3,124.6,124.5,124.4,118.1,118.0,41.3$, $40.8,37.6,29.3,29.1,26.9,26.9,25.5,25.0,23.2,20.9$.

IR: (ATR, neat, $\left.\mathrm{cm}^{-1}\right)$ 3025, 2922, 2881, 2853, 2820, 2229, 1605, 1494, 1453, 1428, 1028, 957, 759, 739

GPC: $(T H F) M w=183.2 \mathrm{kDa}, \mathrm{PDI}=1.851$

DSC: $\left({ }^{\circ} \mathrm{C}, 10^{\circ} \mathrm{C} / \mathrm{min}\right)$ Parent $: T_{g}=105^{\circ} \mathrm{C}$, Functionalized : $T_{g}=107^{\circ} \mathrm{C}$

TGA: $\left({ }^{\circ} \mathrm{C}, 15^{\circ} \mathrm{C} / \mathrm{min}\right) \mathrm{T}_{\mathrm{deg}}=405^{\circ} \mathrm{C}$

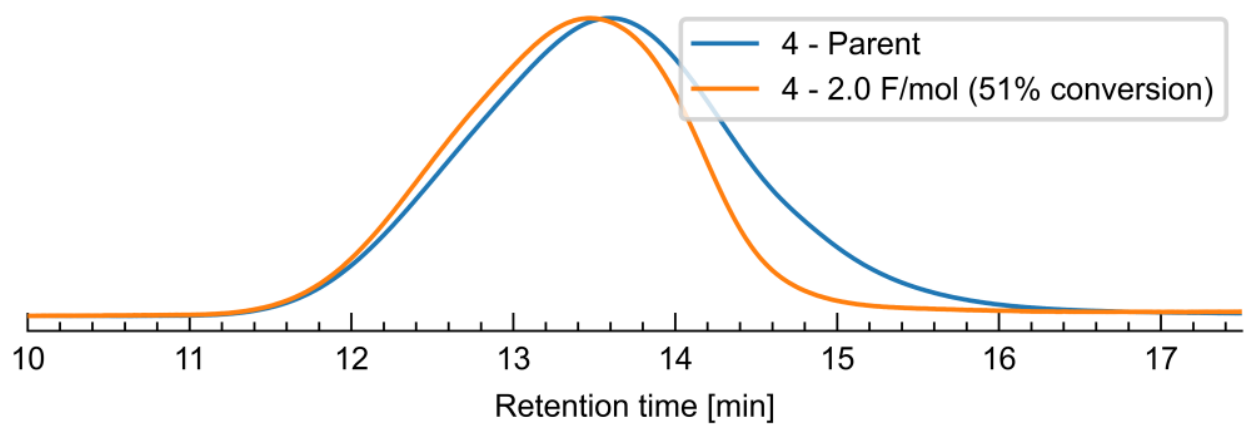

Figure S20. GPC trace of $\mathbf{4}$ overlayed with parent polymer. 

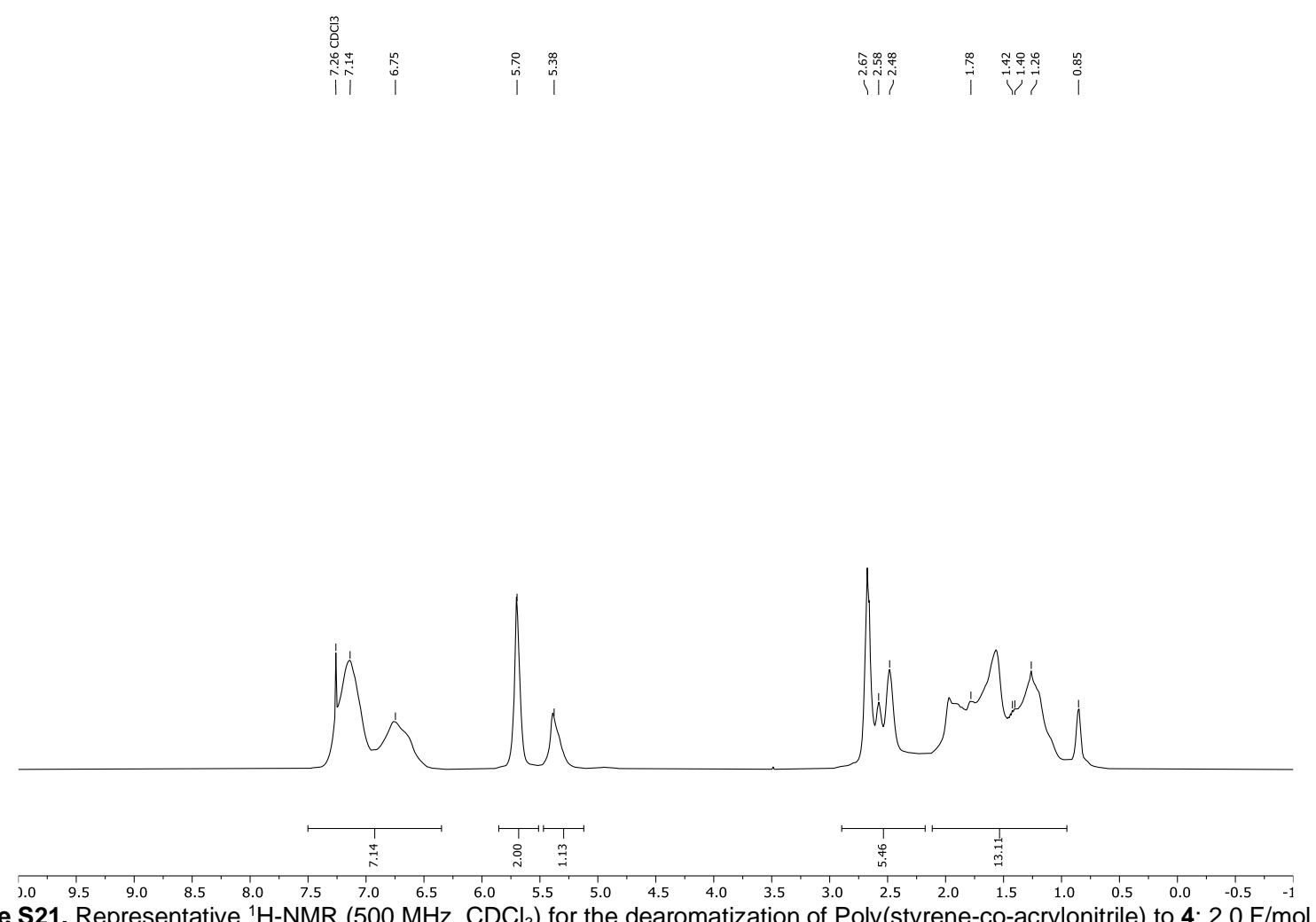

Figure S21. Representative ${ }^{1} \mathrm{H}-\mathrm{NMR}\left(500 \mathrm{MHz}, \mathrm{CDCl}_{3}\right.$ ) for the dearomatization of Poly(styrene-co-acrylonitrile) to 4: $2.0 \mathrm{~F} / \mathrm{mol}$.



Figure S22. Representative ${ }^{13} \mathrm{C}-\mathrm{NMR}\left(126 \mathrm{MHz},{ }^{90} \mathrm{CDCl}_{3}\right)$ for the dearomatization of Poly(styrene-co-acrylonitrile) to $4: 2.0 \mathrm{~F} / \mathrm{mol}$ 


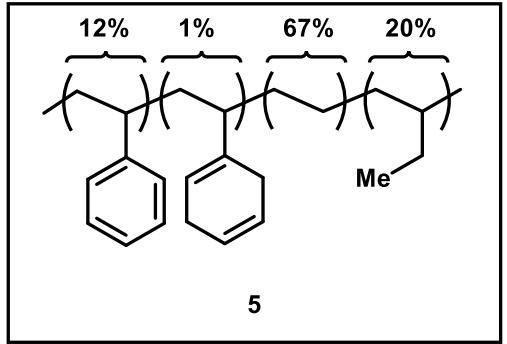

5: Poly(styrene-block-ethylene-ran-butylene-block styrene) $(\mathrm{Mw}=118$ $\mathrm{kDa}$ ) possessing a 1:5:1.5 molar ratio of styrene: ethylene: butylene units, (580 mg, $1.92 \mathrm{mmol}$ of arene repeat unit) was subjected to standard conditions following work-up A. After $2.0 \mathrm{~F} / \mathrm{mol}(5.12 \mathrm{~h}), 10 \%$ conversion to the diene (12\% overall) was observed. Due to large interference in the alkyl region of the ${ }^{1} \mathrm{H}-\mathrm{NMR}$, the degree of overreduction could not be accurately calculated. A ratio of 5:1 diene: overreduced product was applied by analogy to poly(styrene).

${ }^{1}$ H NMR: $\left(500 \mathrm{MHz}, \mathrm{CDCl}_{3}\right) \delta 7.10,7.08,6.58,6.51,6.47,6.38,5.67,5.37,5.26,5.19,2.63,2.44,2.18$, $2.06,1.95,1.86,1.27,1.09,0.85,0.84,0.82$

${ }^{13} \mathrm{C}$ NMR: $\left(126 \mathrm{MHz}, \mathrm{CDCl}_{3}\right) \delta 145.5,128.2,128.1,128.0,127.8,127.6,127.5,125.8,125.6,40.5,39.0$, $38.7,38.5,38.0,36.4,36.3,36.3,36.3,36.2$, 33.8, 33.7, 33.6, 33.5, 33.4, 30.8, 30.5, 30.4, 30.2, 29.9, 29.9, $26.9,26.8,26.8,26.7,26.3,26.2,26.1,26.1,11.0,11.0,10.9,10.8,10.7$.

IR: (ATR, neat, $\left.\mathrm{cm}^{-1}\right)$ 2960, 2922, 2853, 1601, 1493, 1454, 1462, 1379, 906

GPC: (THF) Neither parent nor functionalized material was fully soluble in THF at the desired concentration

DSC: $\left({ }^{\circ} \mathrm{C}, 10^{\circ} \mathrm{C} / \mathrm{min}\right)$ Parent: $T_{g}=-56{ }^{\circ} \mathrm{C}$, Functionalized : $T_{g}=-56{ }^{\circ} \mathrm{C}$

TGA: $\left({ }^{\circ} \mathrm{C}, 15^{\circ} \mathrm{C} / \mathrm{min}\right) \mathrm{T}_{\text {deg }}=427^{\circ} \mathrm{C}$ 


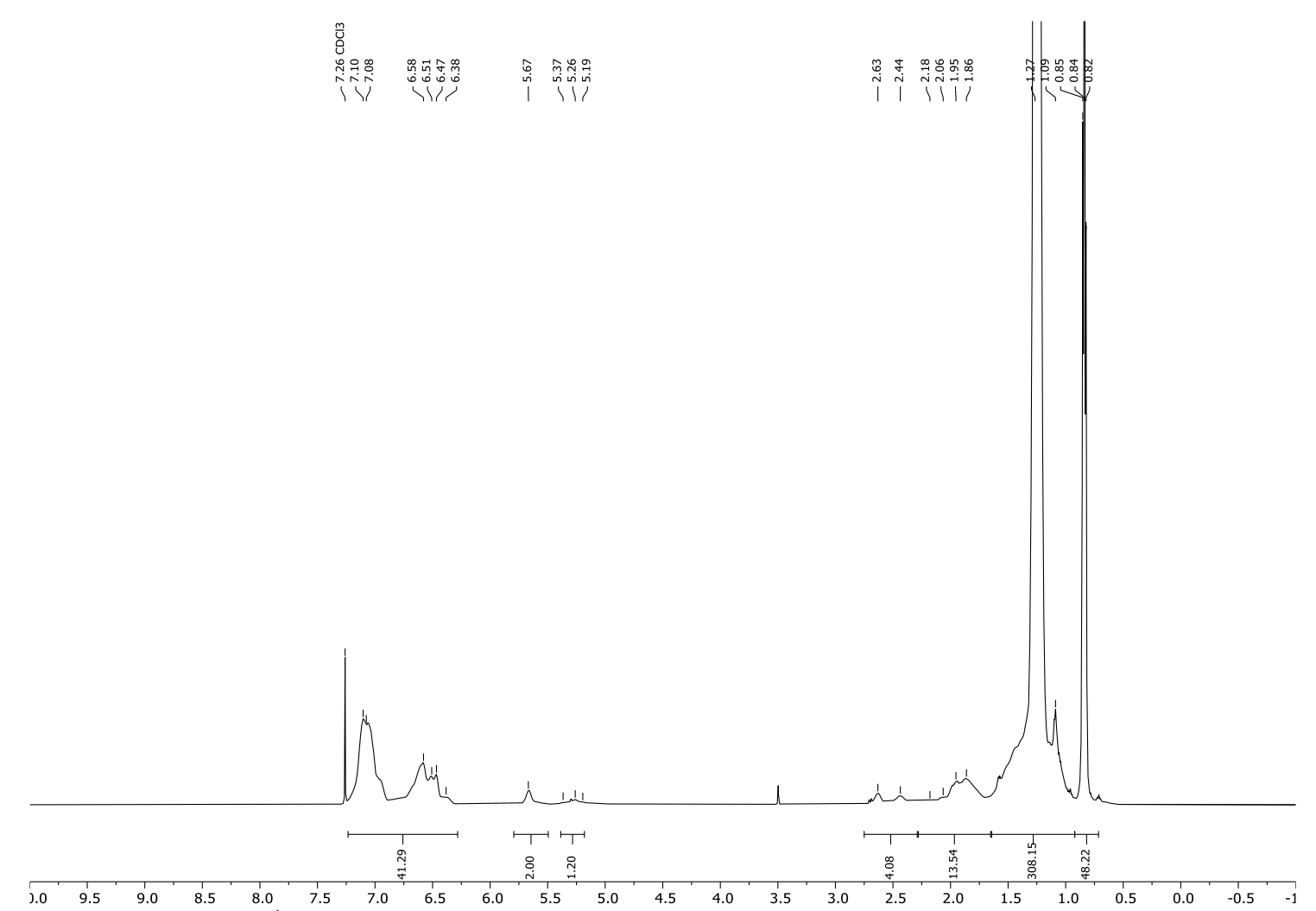

Figure S23. Representative ${ }^{1} \mathrm{H}-\mathrm{NMR}\left(500 \mathrm{MHz}, \mathrm{CDCl}_{3}\right)$ for the dearomatization of Poly(styrene-block-ethylene-ran-butylene-block styrene) to $5: 2.0 \mathrm{~F} / \mathrm{mol}$.


Figure S24. Representative ${ }^{13} \mathrm{C}-\mathrm{NMR}\left(126 \mathrm{MHz}, \mathrm{CDCl}_{3}\right)$ for the dearomatization of Poly(styrene-block-ethylene-ran-butylene-block styrene) to 5: $2.0 \mathrm{~F} / \mathrm{mol}$. 


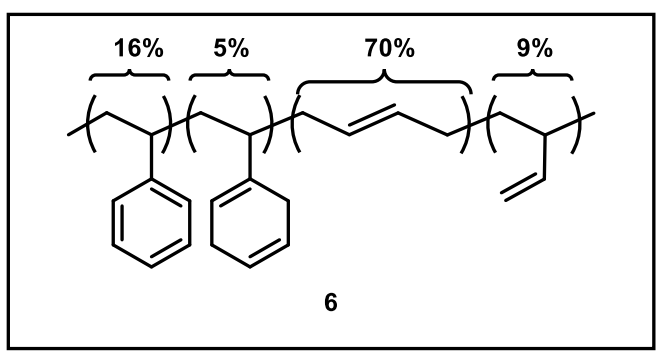

6: Poly(styrene-block-butadiene-block-styrene), possessing a 1:3.3:0.4 molar ratio of styrene:1,4-butiene:1,2-butadiene units, (584 mg, $1.92 \mathrm{mmol}$ of arene repeat unit) was subjected to standard conditions following work-up A. After $2.0 \mathrm{~F} / \mathrm{mol}$ (5.12 h), $22 \%$ conversion to the diene ( $26 \%$ overall) was observed. Due to large interference in the alkyl and olefin region of the ${ }^{1} \mathrm{H}-\mathrm{NMR}$, the degree of overreduction could not be accurately calculated. A ratio of 5:1 diene: overreduced product was applied by analogy to poly(styrene). The bisallylic peaks at $2.62 \mathrm{ppm}$ and $2.43 \mathrm{ppm}$ were used to determine conversion.

${ }^{1}$ H NMR: $\left(500 \mathrm{MHz}, \mathrm{CDCl}_{3}\right) \delta 7.09,7.05,6.57,6.50,6.46,6.38,5.66,5.60,5.59,5.57,5.57,5.55,5.54$, $5.42,5.41,5.38,5.37,5.25,4.98,4.97,4.94,4.92,2.62,2.43,2.09,2.04,1.44,1.34,1.30,1.26$

${ }^{13} \mathrm{C}$ NMR: $\left(126 \mathrm{MHz}, \mathrm{CDCl}_{3}\right) \delta 142.9,142.8,130.3,130.3,130.3,130.3,130.2,130.2,130.2,130.1,130.1$, 130.0, 129.8, 129.8, 129.8, 129.7, 129.6, 129.6, 129.6, 129.4, 128.1, 127.8, 125.8, 114.5, 114.4, 43.7, 38.3, $38.3,34.3,34.1,32.9,32.9,32.8,30.4,30.3,29.9,29.8,27.7,27.6,27.4,25.1,25.0$

GPC: Not performed due to insolubility of parent and functionalized material at the required concentration in THF

IR: (ATR, neat, $\left.\mathrm{cm}^{-1}\right)$ 3005, 2918, 2846, 1641, 1601, 1493, 1451, 1028, 993, 964, 911, 757, 747, 727

DSC: $\left({ }^{\circ} \mathrm{C}, 10{ }^{\circ} \mathrm{C} / \mathrm{min}\right)$ Parent: $T_{g 1}=-94^{\circ} \mathrm{C} T_{g 2}=94^{\circ} \mathrm{C}$, Functionalized : $T_{g 1}=-94^{\circ} \mathrm{C} T_{g 2}=92{ }^{\circ} \mathrm{C}$

TGA: $\left({ }^{\circ} \mathrm{C}, 15^{\circ} \mathrm{C} / \mathrm{min}\right) \mathrm{T}_{\text {deg }}=376^{\circ} \mathrm{C}$ 


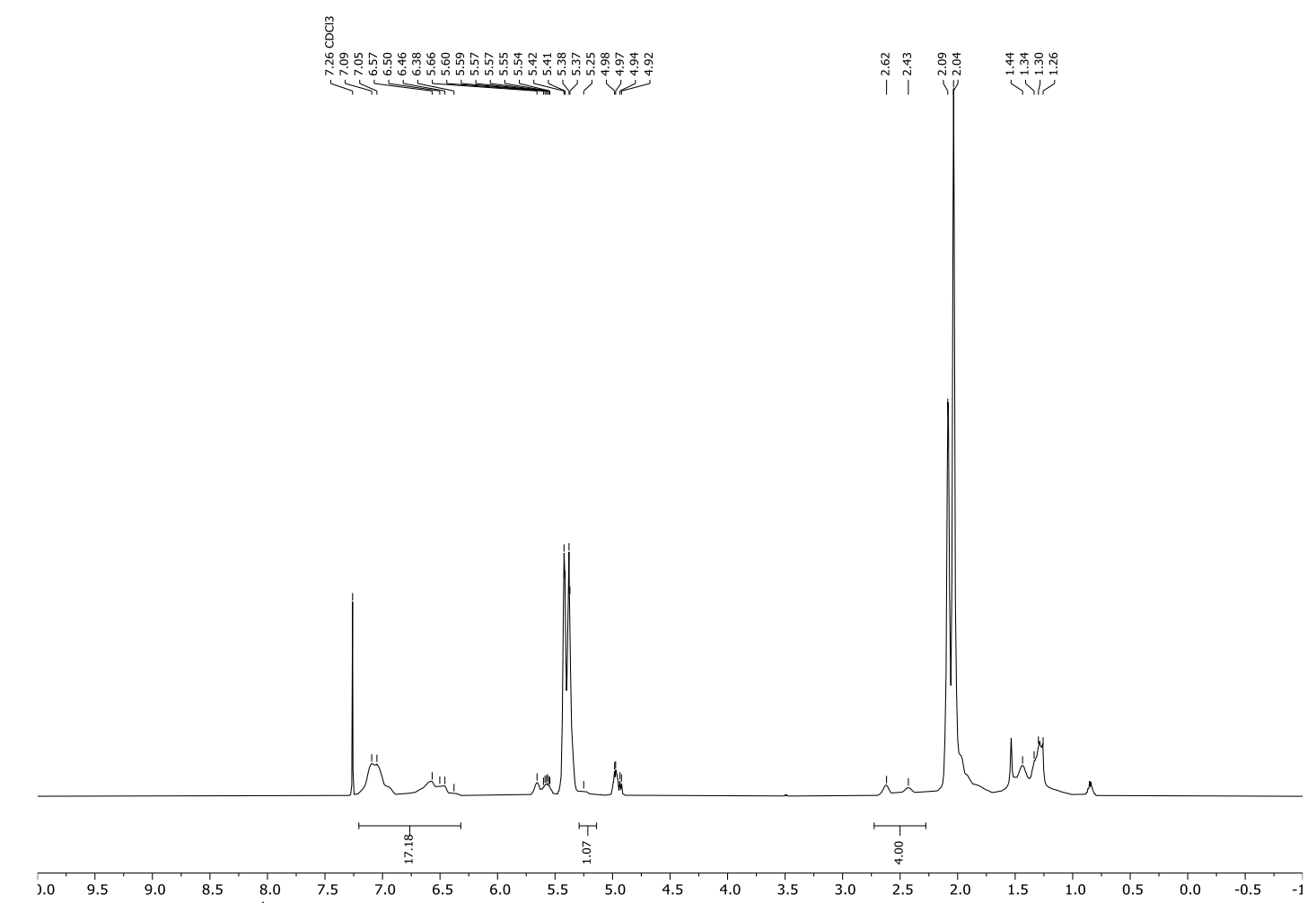

Figure S25. Representative ${ }^{1} \mathrm{H}-\mathrm{NMR}\left(500 \mathrm{MHz}, \mathrm{CDCl}_{3}\right)$ for the dearomatization of Poly(styrene-block-butadiene-block-styrene) to 6: 2.0 F/mol.
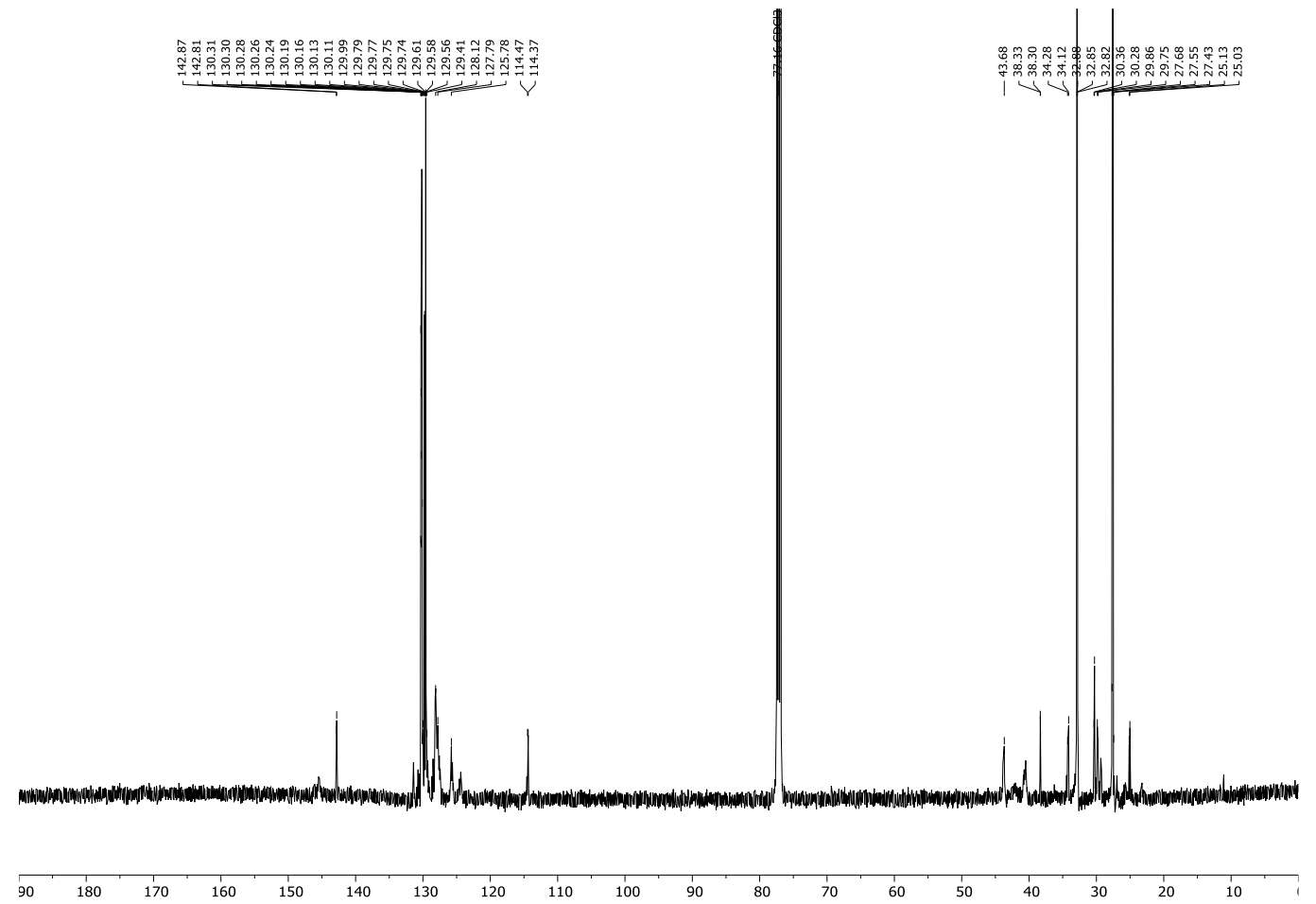

Figure S26. Representative ${ }^{13} \mathrm{C}-\mathrm{NMR}\left(126 \mathrm{MHz}, \mathrm{CDCl}_{3}\right)$ for the dearomatization of Poly(styrene-block-butadiene-block-styrene) to 6: $2.0 \mathrm{~F} / \mathrm{mol}$. 


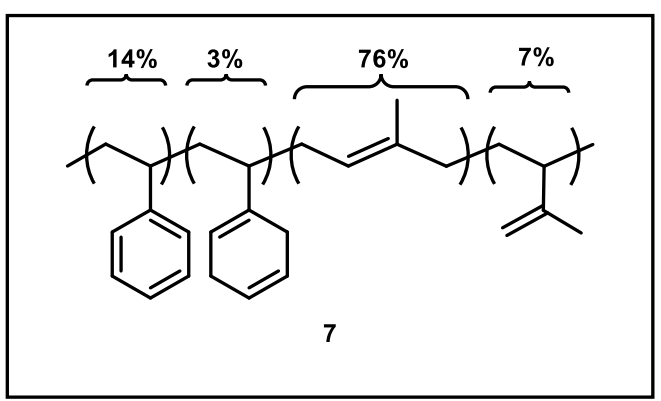

7: Poly(styrene-block-isoprene-block-styrene), possessing a 1:4.4:0.4 molar ratio of styrene:1,4-isoprene:1,2-isoprene units, (828 mg, $1.92 \mathrm{mmol}$ of arene repeat unit) was subjected to standard conditions following work-up A. After $2.0 \mathrm{~F} / \mathrm{mol}$ $(5.12 \mathrm{~h}), 17 \%$ conversion to the diene ( $20 \%$ overall) was observed. Due to large interference in the alkyl and olefin region of the ${ }^{1} \mathrm{H}-\mathrm{NMR}$, the degree of overreduction could not be accurately calculated. A ratio of 5:1 diene : overreduced product was applied by analogy to poly(styrene).

${ }^{1}$ H NMR: $\left(500 \mathrm{MHz}, \mathrm{CDCl}_{3}\right) \delta 7.10,7.05,6.61,6.50,6.46,5.66,5.14,5.13,5.11,5.10,5.08,4.76,4.74$, $4.68,4.66,2.63,2.43,2.05,1.99,1.98,1.68,1.66,1.63,1.61,1.54$.

${ }^{13} \mathrm{C}$ NMR: $\left(126 \mathrm{MHz}, \mathrm{CDCl}_{3}\right) \delta$ 147.7, 135.5, 135.4, 135.3, 135.3, 135.1, 135.0, 128.1, 125.8, 125.3, 125.2, 125.2, 125.1, 125.1, 124.7, 124.5, 124.4, 124.4, 124.4, 124.3, 123.9, 123.2, 111.6, 111.5, 48.1, 47.9, 47.6, 40.2 , 40.2, 40.2, 39.9, 37.7, 32.5, 32.4, 32.4, 32.2, 32.2, 32.1, 31.4, 31.4, 30.0, 26.9, 26.8, 26.8, 26.7, 26.6, $26.6,26.6,26.5,26.5,25.8,23.6,23.6,18.8,18.7,16.3,16.2,16.1,16.1$

GPC: Not performed due to insolubility of parent and functionalized material at the required concentration in THF

IR: (ATR, neat, $\left.\mathrm{cm}^{-1}\right)$ 2961, 2919, 2852, 1671, 1651, 1605, 1492, 1450, 1375, 1090, 1029, 888, 836, 758

DSC: $\left({ }^{\circ} \mathrm{C}, 10^{\circ} \mathrm{C} / \mathrm{min}\right)$ Parent: $T_{g}=-62^{\circ} \mathrm{C}$, Functionalized: $T_{g}=-62^{\circ} \mathrm{C}$

TGA: $\left({ }^{\circ} \mathrm{C}, 15^{\circ} \mathrm{C} / \mathrm{min}\right) \mathrm{T}_{\text {deg }}=367^{\circ} \mathrm{C}$ 


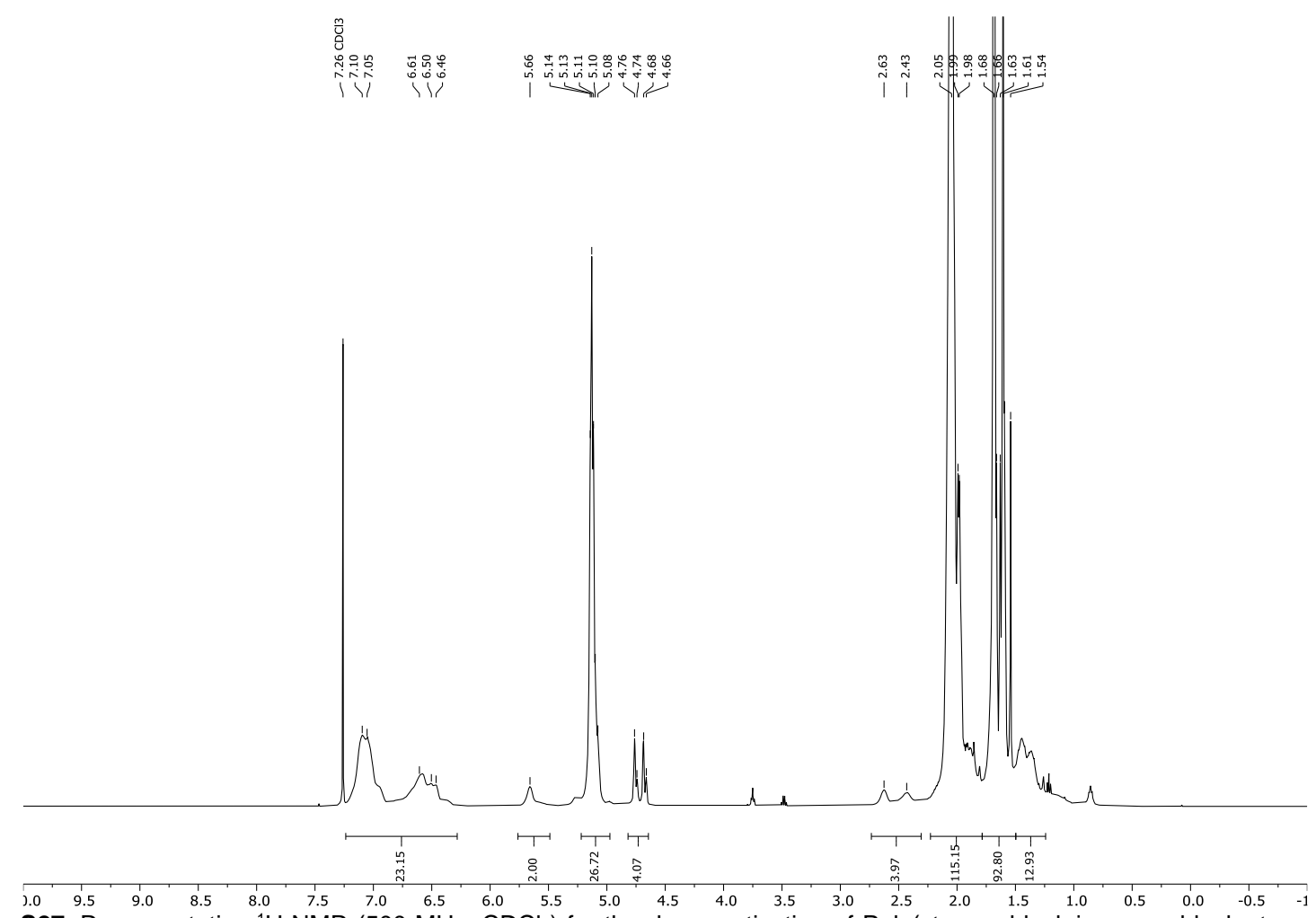

Figure S27. Representative ${ }^{1} \mathrm{H}-\mathrm{NMR}\left(500 \mathrm{MHz}, \mathrm{CDCl}_{3}\right)$ for the dearomatization of Poly(styrene-block-isoprene-block-styrene) to 7: 2.0 F/mol.

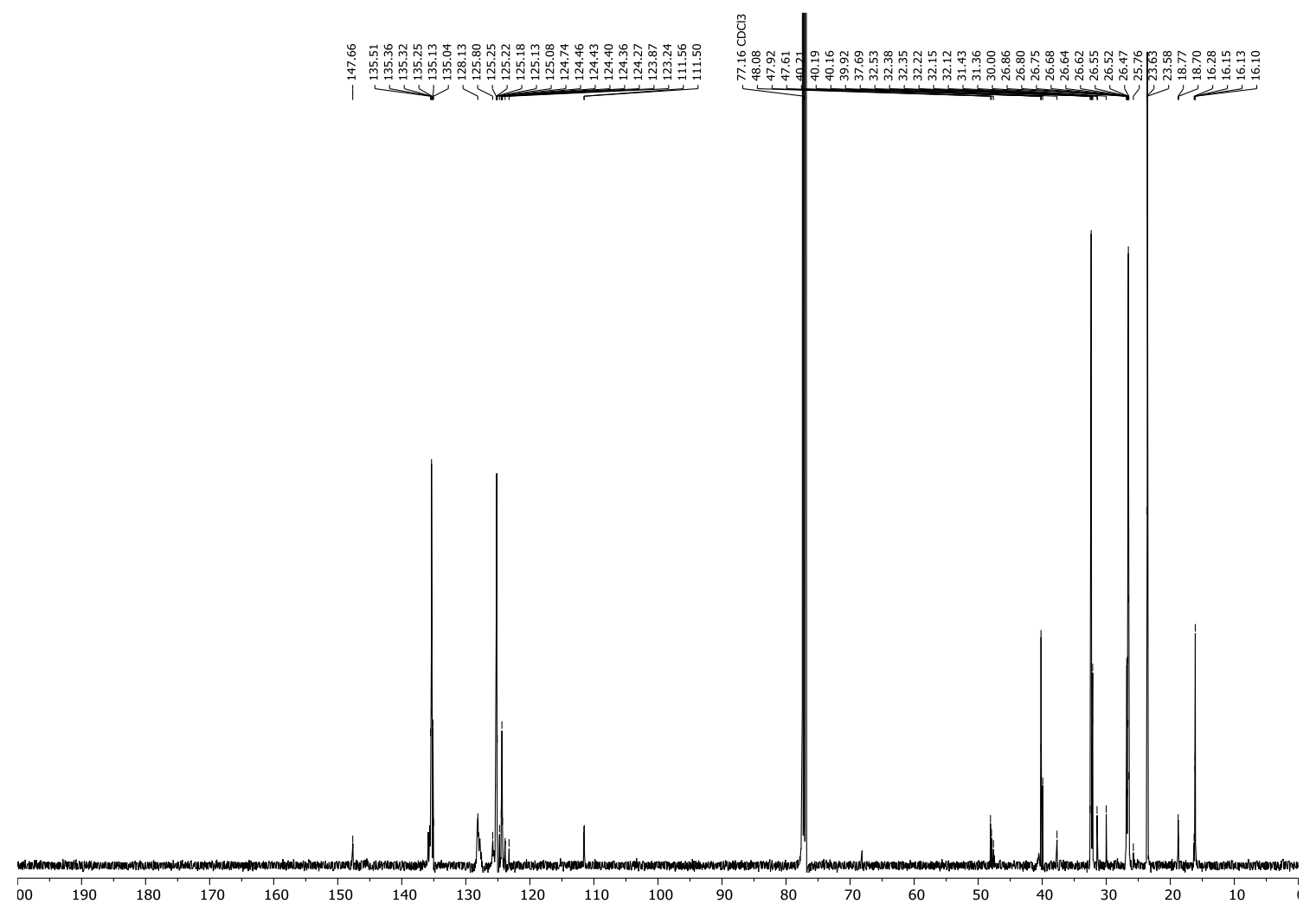

Figure S28. Representative ${ }^{13} \mathrm{C}-\mathrm{NMR}\left(126 \mathrm{MHz} \mathrm{CDCl}_{3}\right)$ for the dearomatization of Poly(styrene-block-isoprene-block-styrene) to 7: 2.0 F/mol. 
3-4. Table S3. Failed substrates with attempted modifications.

Substrate<smiles>CC(C)CC(c1ccccc1)(C(C)C)C(C)(C)C</smiles>

8: poly(a-methystyrene)

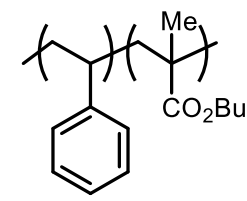

9: poly(styrene-co-Bu-acrylate)<smiles>CC(C)(C)CC(c1ccccc1)C(C)(C)C1c2ccccc2-c2ccccc21</smiles>

10: syndiotactic PS<smiles>CC(C)(C)OCCOC(=O)c1ccc(C(C)(C)C)cc1</smiles>

11: poly(ethylene terephthalate)
Observation

Reaction proceeded a significant degree of byproduct formation. No decomposition was observed by SEC.

A significant degree of cross-linking was observed, rendered us incapable

of analyzing the product outcome

in quantifiable manner.
Attempted modifications

Performing reaction at $-78^{\circ} \mathrm{C}$.

Increasing/decreasing current density.

Performing reaction at $-78^{\circ} \mathrm{C}$. Decreasing polymer concentration to $0.05 \mathrm{M}$ Decreasing current density.

Performing the reaction at reflux. Decreasing polymer concentration Decreasing HMPA loading to 1.0 equiv.

Complete insolubility

Addition of HFIP or TFA in a 10:1 ratio with THF Performing reaction at reflux 


\section{3-5. Large Scale Procedures}

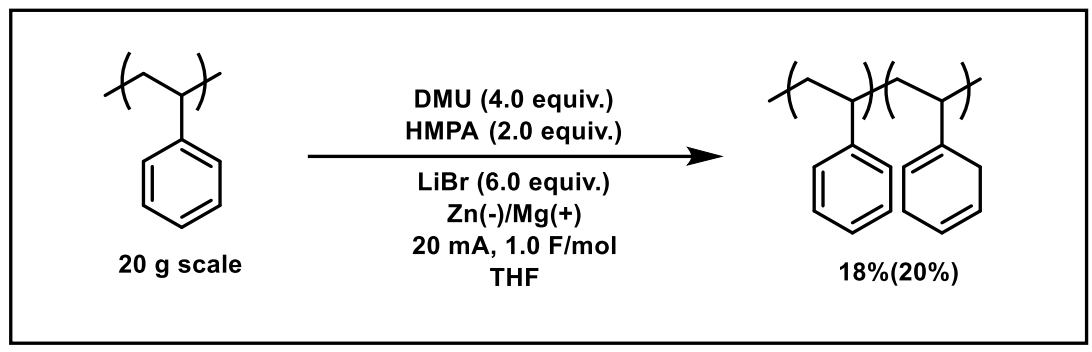

The large-scale reactor was assembled (see Figure S2) and transferred into the glovebox. To the $1 \mathrm{~L}$ media bottle was added poly(styrene) (20 g, $192 \mathrm{mmol}, 1.0$ equiv.), dimethylurea (68 g, $768 \mathrm{mmol}$, 4.0 equiv.), and lithium bromide (100 g, $1.15 \mathrm{~mol}, 6.0$ equiv.). The reaction vessel was screwed shut and brought out of the glovebox. Tetrahydrofuran $(800 \mathrm{~mL})$ and hexamethylphosphoramide $(69 \mathrm{~g}, 67 \mathrm{~mL}, 384 \mathrm{mmol}, 2.0$ equiv.) were added by means of canula and the reaction was stirred until complete dissolution of reagents (approximately 30 minutes). DC power was attached to the vessel by means of alligator clips, and 400 milliamps of current was applied. The reaction was run with high stirring $(600 \mathrm{rpm})$ at room temperature for the allotted reaction time $(1.0 \mathrm{~F} / \mathrm{mol}, 12.8 \mathrm{~h})$.

Work-up: The DC power supply was turned off and the reaction mixture was poured into a stirring solution of $\mathrm{HCl}$ in methanol $(3 \mathrm{~L}, 0.05 \mathrm{M}$, prepared from aqueous $37 \% \mathrm{HCl})$. The reaction vessel and electrodes were washed with DCM (approximately $50 \mathrm{~mL}$ ), which was then added to the beaker containing methanol and crude product. This mixture was stirred for 5 minutes, filtered using a sintered filter funnel, and dried under a blanket of nitrogen for 1 hour. The filter cake containing crude product was redissolved in DCM $(100 \mathrm{~mL})$, stirred for 20 minutes until complete dissolution, poured into a stirring solution of methanol $(2 \mathrm{~L})$, and the precipitate filtered and dried under a flow of nitrogen for 2 hours. The filter cake (now pure product) was collected and dried under vacuum at room temperature for 12 hours to yield the pure product ( $18 \%$ conversion to diene, $20 \%$ overall conversion, $181 \mathrm{kDa}, \mathrm{PDI}=2.76$ ). The product was stored under nitrogen in a $-20^{\circ} \mathrm{C}$ freezer. 


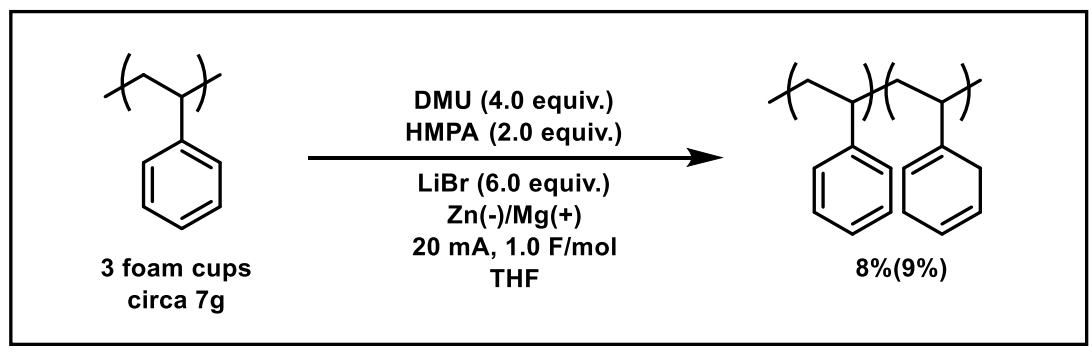

The large-scale reactor was assembled (see Figure S2) and transferred into the glovebox. To the $1 \mathrm{~L}$ media bottle was added poly(styrene) ( 3 foam cups, $7.0 \mathrm{~g}, 274 \mathrm{kDa}, \mathrm{PDI}=3.1,66.8 \mathrm{mmol}$, 1.0 equiv.), dimethylurea (23.6 g, $267 \mathrm{mmol}, 4.0$ equiv.), and lithium bromide ( $34.8 \mathrm{~g}, 401 \mathrm{mmol}, 6.0$ equiv.). The reaction vessel was screwed shut and brought out of the glovebox. Tetrahydrofuran $(600 \mathrm{~mL})$ and hexamethylphosphoramide $(24.0 \mathrm{~g}, 23.3 \mathrm{~mL}, 134 \mathrm{mmol}, 2.0$ equiv.) were added by means of canula and the reaction was stirred until complete dissolution of reagents (approximately 15 minutes). DC power was attached to the vessel by means of alligator clips, and 200 milliamps of current was applied. The reaction was run with strong stirring (600 rpm) at room temperature for the allotted reaction time $(1.0 \mathrm{~F} / \mathrm{mol}, 8.9 \mathrm{~h})$.

Work-up: The DC power supply was turned off and the the reaction mixture was poured into a stirring solution of $\mathrm{HCl}$ in methanol $(2 \mathrm{~L}, 0.05 \mathrm{M}$, prepared from aqueous $37 \% \mathrm{HCl})$. The reaction vessel and electrodes were washed with DCM (approximately $50 \mathrm{~mL}$ ) which was then added to the beaker containing methanol and crude product. This mixture was stirred for approximately 5 minutes, filtered using a sintered filter funnel, and dried under a blanket of nitrogen. The filter cake containing crude product was redissolved in DCM (100 mL), stirred until complete dissolution, poured into a stirring solution of methanol $(1 \mathrm{~L})$, and the precipitate was filtered using a sintered filter funnel and dried under a flow of nitrogen for 2 hours. The filter cake (now pure product) was collected and dried under vacuum at room temperature for 12 hours to yield pure product $(8 \%$ conversion to diene, $9 \%$ overall conversion, $259 \mathrm{kDa}, \mathrm{PDI}=3.3)$. The product was stored under nitrogen in $\mathrm{a}-20^{\circ} \mathrm{C}$ freezer. 


\section{3-6. Varying Mw Experiment}<smiles>CC(C)(C)CC(c1ccccc1)C(C)(C)C</smiles>

varying $\mathrm{Mw}$

$\mathrm{PDI}<1.03$

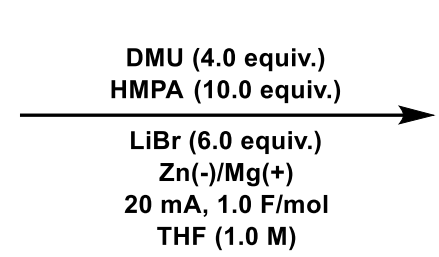

(1)

Monodisperse poly(styrene) standards were obtained from commercial vendors and subjected to standard reaction conditions following work-up $A$. The reaction was halted after $1.0 \mathrm{~F} / \mathrm{mol}$ and the conversion was recorded, the results are tabulated below:

\begin{tabular}{cccc} 
Mw $(\mathrm{kDa})$ & $\mathrm{Ln}(\mathrm{Mw})$ & Conversion to diene & Total Conversion \\
\hline 4.0 & 8.29 & 0.32 & 0.39 \\
6.1 & 8.72 & 0.31 & 0.35 \\
12.0 & 9.39 & 0.27 & 0.31 \\
19.5 & 9.87 & 0.26 & 0.30 \\
63.0 & 11.05 & 0.17 & 0.20 \\
100 & 11.51 & 0.15 & 0.19 \\
200 & 12.20 & 0.12 & 0.14 \\
400 & 12.90 & 0.10 & 0.11 \\
1,800 & 14.4 & 0.07 & 0.08
\end{tabular}

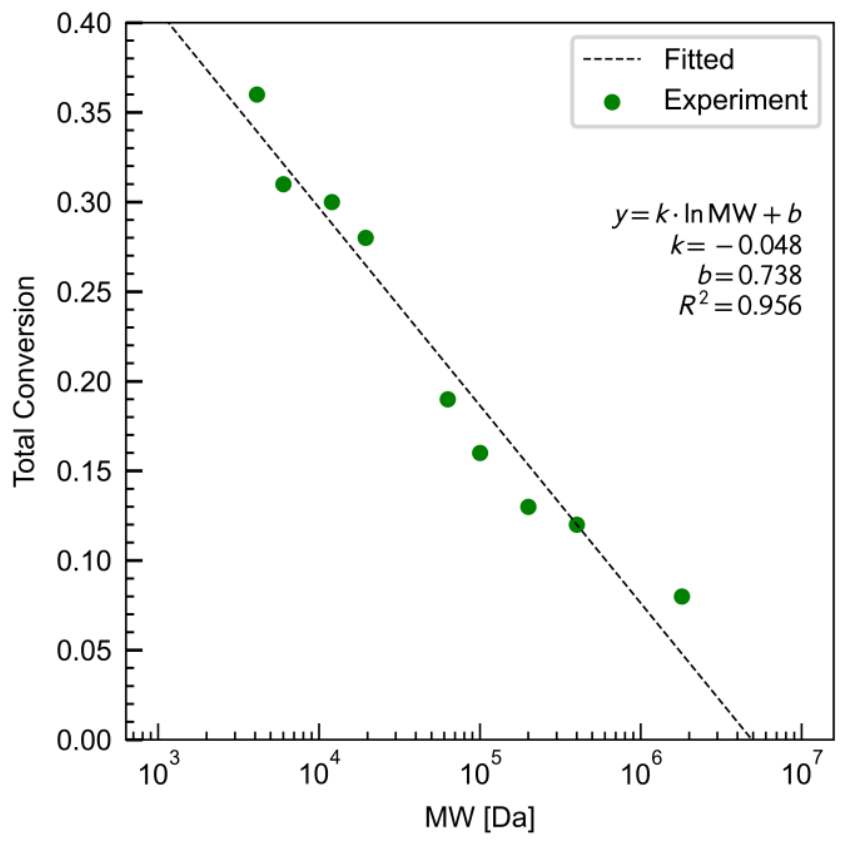

Figure S29. Top: Tabulated raw data. Bottom: Plot of MW vs. Conversion at 1.0 F/mol. 


\section{3-7. Derivatization}

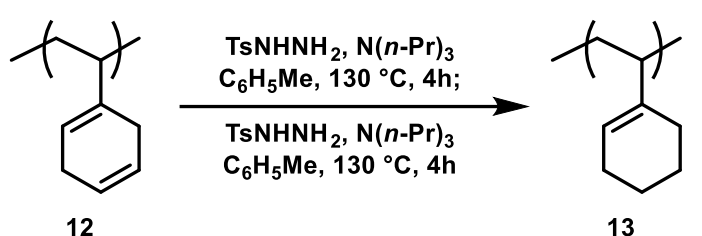

Diimide reduction of Poly(vinylcyclohexadiene): To an oven-dried $25 \mathrm{~mL}$ round bottom was added 12 (120 $\mathrm{mg}, 0.9 \mathrm{mmol}$ of diene units), tosylhydrazide (537 mg, $2.9 \mathrm{mmol}, 3.0$ equiv.), $n-\mathrm{Pr}_{3} \mathrm{~N}$ (1.1 mL, $826 \mathrm{mg}, 5.8$ $\mathrm{mmol}, 6.0$ equiv.), and toluene $(9.6 \mathrm{~mL})$. The solution was sparged with $\mathrm{N}_{2}$ for 20 minutes, equipped with a condenser, and then heated to reflux for two hours (external bath temperature was set to $130{ }^{\circ} \mathrm{C}$ ). The solution became homogenous at approximately $90^{\circ} \mathrm{C}$, and $\mathrm{N}_{2}$ evolution was noted at approximately 100 ${ }^{\circ} \mathrm{C}$. Afterwards the reaction was cooled to room temperature, at which point tosylhydrazide (179 $\mathrm{mg}, 0.97$ mmol, 1.0 equiv. $), \mathrm{N}(n-\operatorname{Pr})_{3}(0.367 \mathrm{~mL}, 275 \mathrm{mg}, 1.93 \mathrm{mmol}, 2.0$ equiv.) were added and the reaction was heated to reflux for another two hours. Afterwards the reaction was cooled to room temperature, poured into cold methanol, and filtered. The filter cake containing crude product was re-dissolved in DCM (5 mL) and precipitated once more from methanol and filtered to yield pure 13. The product was collected and dried under vacuum at room temperature for 4 hours prior to analysis. The desired compound was obtained with complete conversion of diene sub-units.

${ }^{1}$ H NMR: $\left(500 \mathrm{MHz}, \mathrm{CDCl}_{3}\right) \delta$ 7.19, 7.10, 7.01, 5.67, 5.29, 5.22, 5.06, 2.64, 2.36, 1.95, 1.76, 1.55, 1.13.

${ }^{13} \mathrm{C}$ NMR: $\left(126 \mathrm{MHz}, \mathrm{CDCl}_{3}\right) \delta$ 140.0, 138.3, 127.9, 123.4, 122.3, 121.9, 42.6, 38.2, 27.4, 25.6, 25.6, 24.3, $23.8,23.5,23.3$

IR: (ATR, neat, $\left.\mathrm{cm}^{-1}\right)$ 2920, 2853, 2833, 1447, 1337, 1305, 1268, 1136, 918, 801, 760

GPC (THF) Mw $=152 \mathrm{kDa}, \mathrm{PDI}=2.14$

TGA: $\left({ }^{\circ} \mathrm{C}, 15^{\circ} \mathrm{C} / \mathrm{min}\right) \mathrm{T}_{\text {deg }}=378^{\circ} \mathrm{C}$

DSC: $\left({ }^{\circ} \mathrm{C}, 10^{\circ} \mathrm{C} / \mathrm{min}\right) \mathrm{T}_{\mathrm{g}}=107^{\circ} \mathrm{C}$ 




Figure S30. Representative ${ }^{1} \mathrm{H}-\mathrm{NMR}\left(500 \mathrm{MHz}, \mathrm{CDCl}_{3}\right)$ for the diimide reduction of Poly(vinylcyclohexadiene) (12) to 13. 




To a stirring solution of 13 (90 $\mathrm{mg}, 0.78 \mathrm{mmol}$ of tertiary olefin units) in toluene $(0.1 \mathrm{M})$ at $0{ }^{\circ} \mathrm{C}$, was slowly added mCPBA (54 mg, $0.23 \mathrm{mmol}, 0.3$ equiv. with respect to tertiary olefin units). The solution was slowly warmed to room temperature over $1 \mathrm{~h}$ and stirred for another three hours. The solvent was concentrated to a third of the original volume, and methanol was added to precipitate the polymer. The solution was filtered and the filtrate was dried on a fritted funnel. The crude polymer was collected, dissolved in DCM (1 $\mathrm{mL}$ ), and precipitated from methanol once more. The polymer was collected and dried under vacuum for four hours prior to analysis. The desired compound was obtained with $28 \%$ conversion of the tertiary olefin sub-units, as determined through conversion in the ${ }^{1} \mathrm{H}-\mathrm{NMR}$.

${ }^{1} \mathrm{H}$ NMR: $\left(600 \mathrm{MHz}, \mathrm{CDCl}_{3}\right) \delta$ 7.21, 7.10, 7.03, 5.28, 5.10, 2.89, 1.96, 1.76, 1.54, 1.41, 1.25, 1.21, 0.89

${ }^{13} \mathrm{C}$ NMR: $\left(151 \mathrm{MHz}, \mathrm{CDCl}_{3}\right) \delta$ 140.0, 138.1, 128.4, 128.0, 125.4, 123.6, 62.4, 42.5, 41.0, 38.3, 27.2, 25.6, 23.3, 20.6, 20.1.

IR: (ATR, neat, $\left.\mathrm{cm}^{-1}\right)$ 2920, 2853, 1447, 1339, 1305, 1136, 918, 851

GPC (THF) Mw $=152.0 \mathrm{kDa}, \mathrm{PDI}=2.45$

DSC: $\left({ }^{\circ} \mathrm{C}, 10^{\circ} \mathrm{C} / \mathrm{min}\right) \mathrm{T}_{\mathrm{g}}=120^{\circ} \mathrm{C}$

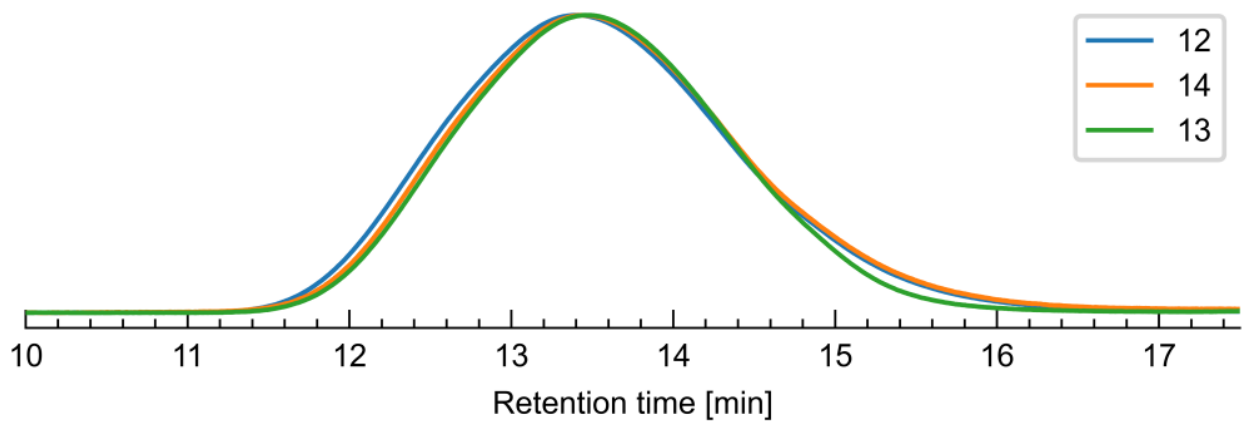

Figure S31. GPC traces of 12, 13, and 14. 


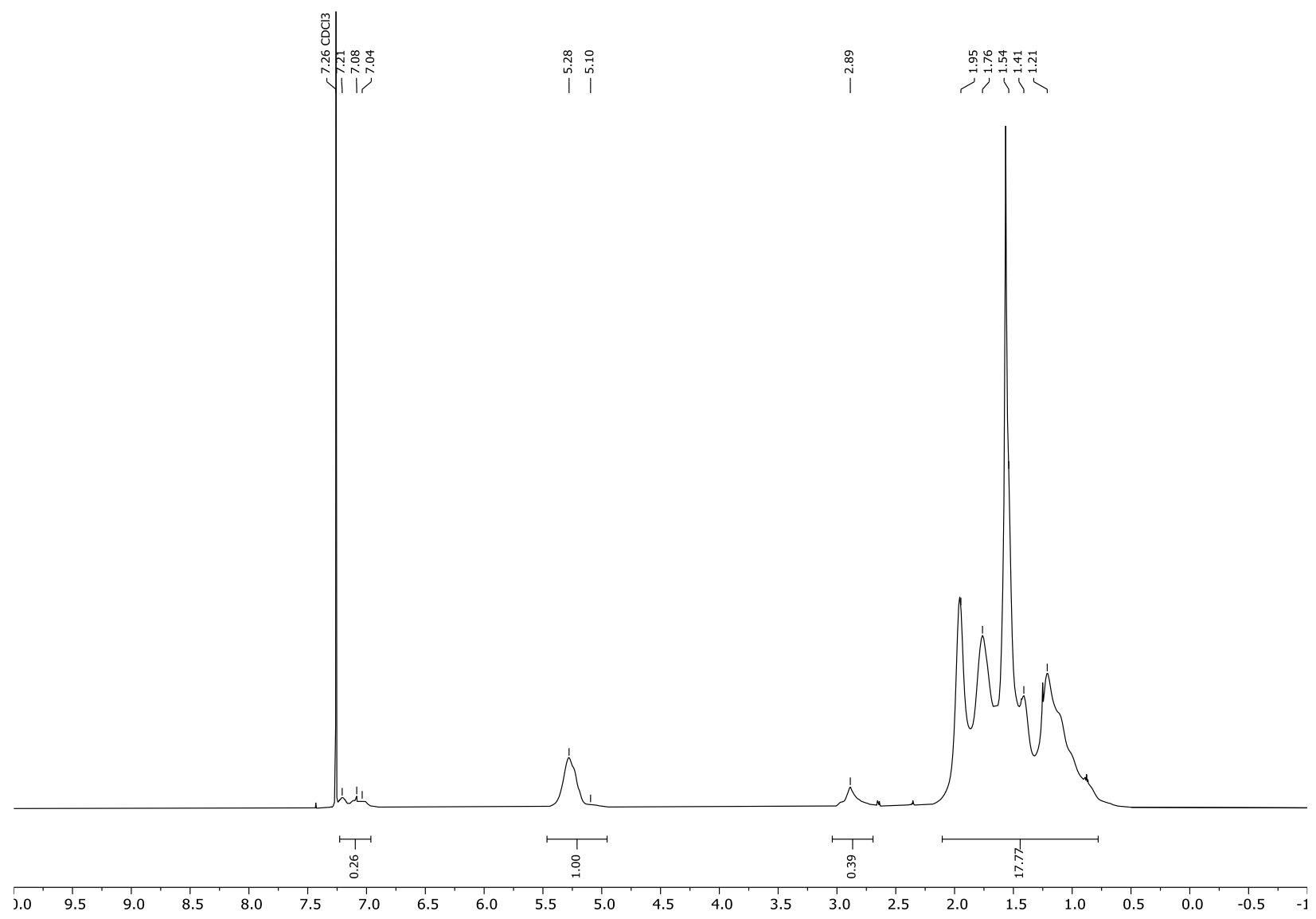

Figure S32. Representative ${ }^{1} \mathrm{H}-\mathrm{NMR}\left(600 \mathrm{MHz}, \mathrm{CDCl}_{3}\right)$ for the epoxidation of $\mathbf{1 3}$ to $\mathbf{1 4}$. 


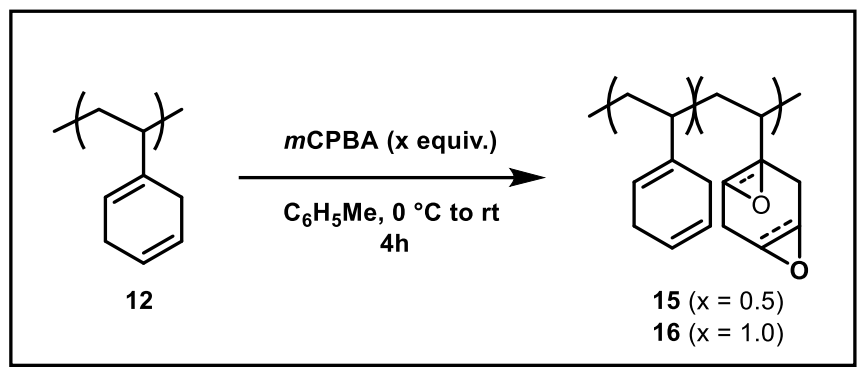

To a stirring solution of 12 (50 mg scale, $0.78 \mathrm{mmol}$ of tertiary olefin units) in toluene $(0.1 \mathrm{M})$ at $0{ }^{\circ} \mathrm{C}$, was slowly added $m$ CPBA. The solution was slowly warmed to room temperature over $1 \mathrm{~h}$ and stirred for another three hours. The solvent was concentrated to a third of the original volume, and methanol was added to precipitate the polymer. The solution was filtered and dried on a frit. The crude polymer was collected, dissolved in DCM $(1 \mathrm{~mL})$, and precipitated from methanol once more. The polymer was collected and dried under vacuum for four hours. Due to excessive peak broadening in both the alkyl and epoxide region, accurate determination of epoxidation conversion could not be assessed by ${ }^{1} \mathrm{H}-\mathrm{NMR}$, thus near-quantitative epoxidation of olefins was assumed.

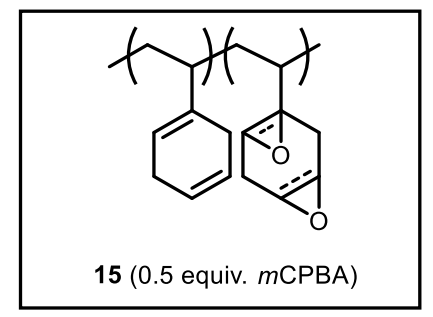

15 was obtained following the general procedure utilizing $52 \mathrm{mg}(0.22 \mathrm{mmol})$ of mCPBA.

${ }^{1} \mathrm{H}$ NMR: $\left(600 \mathrm{MHz}, \mathrm{CDCl}_{3}\right) \delta 7.18,5.67,5.44,3.20,3.06,2.44,2.32,1.91,1.41,1.19$.

${ }^{13} \mathrm{C}$ NMR: $\left(151 \mathrm{MHz}, \mathrm{CDCl}_{3}\right) \delta 146.3,140.00,138.1,128.0,125.4,123.6,62.4,42.5,41.0,27.2,25.6$, 23.3, 20.1 .

IR: (ATR, neat, $\left.\mathrm{cm}^{-1}\right)$ 3026, 2993, 2921, 2823, 1450, 1420, 1349, 1227, 1179, 947, 862

GPC (THF) Mw $=164.2 \mathrm{kDa}, \mathrm{PDI}=2.33$

DSC: $\left({ }^{\circ} \mathrm{C}, 10^{\circ} \mathrm{C} / \mathrm{min}\right) \mathrm{T}_{\mathrm{g}}=130^{\circ} \mathrm{C}$ 


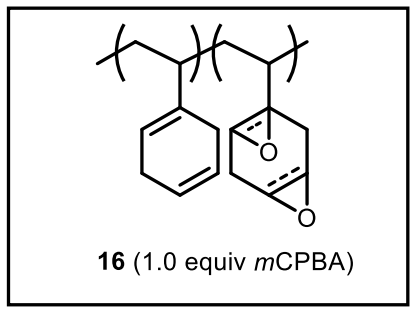

16 was obtained following the general procedure utilizing $103 \mathrm{mg}(0.45$ mmol) of $m$ CPBA.

${ }^{1}$ H NMR: $\left(600 \mathrm{MHz}, \mathrm{CDCl}_{3}\right) \delta 7.12,5.65,5.42,5.30,3.18,2.97,2.63,2.44,1.93,1.43,1.18$

${ }^{13} \mathrm{C}$ NMR: $\left(151 \mathrm{MHz}, \mathrm{CDCl}_{3}\right) \delta 128.4,124.3,121.4,61.1,50.9,40.3,25.9,24.0,19.8$.

IR: (ATR, neat, $\left.\mathrm{cm}^{-1}\right)$ 3025, 292, 2818, 1450, 1421, 1347, 1228, 957, 862

GPC (THF) Mw $=158.4 \mathrm{kDa}, \mathrm{PDI}=2.26$

DSC: $\left({ }^{\circ} \mathrm{C}, 10^{\circ} \mathrm{C} / \mathrm{min}\right) \mathrm{T}_{\mathrm{g}}=162{ }^{\circ} \mathrm{C}$

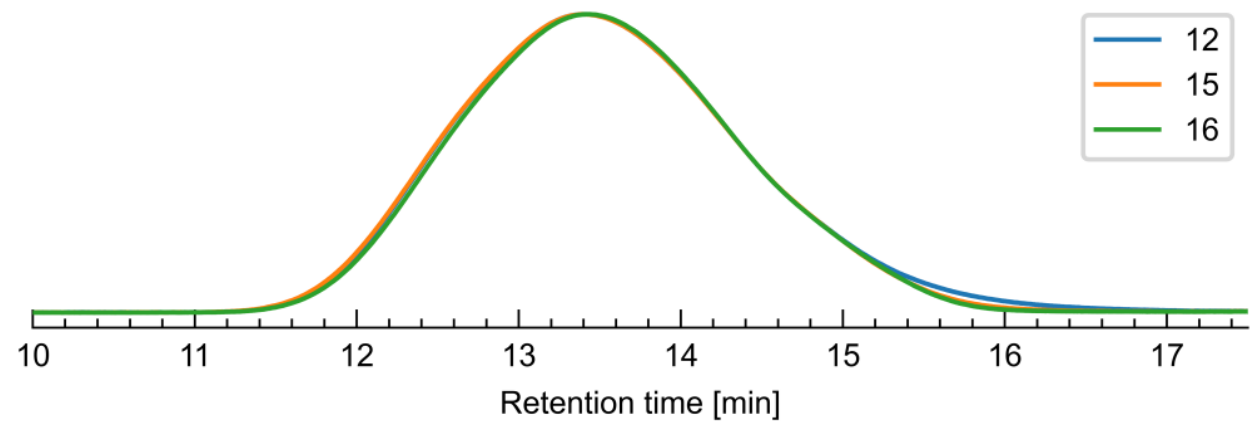

Figure S33. GPC trace of 15 and 16 overlayed with 12. 

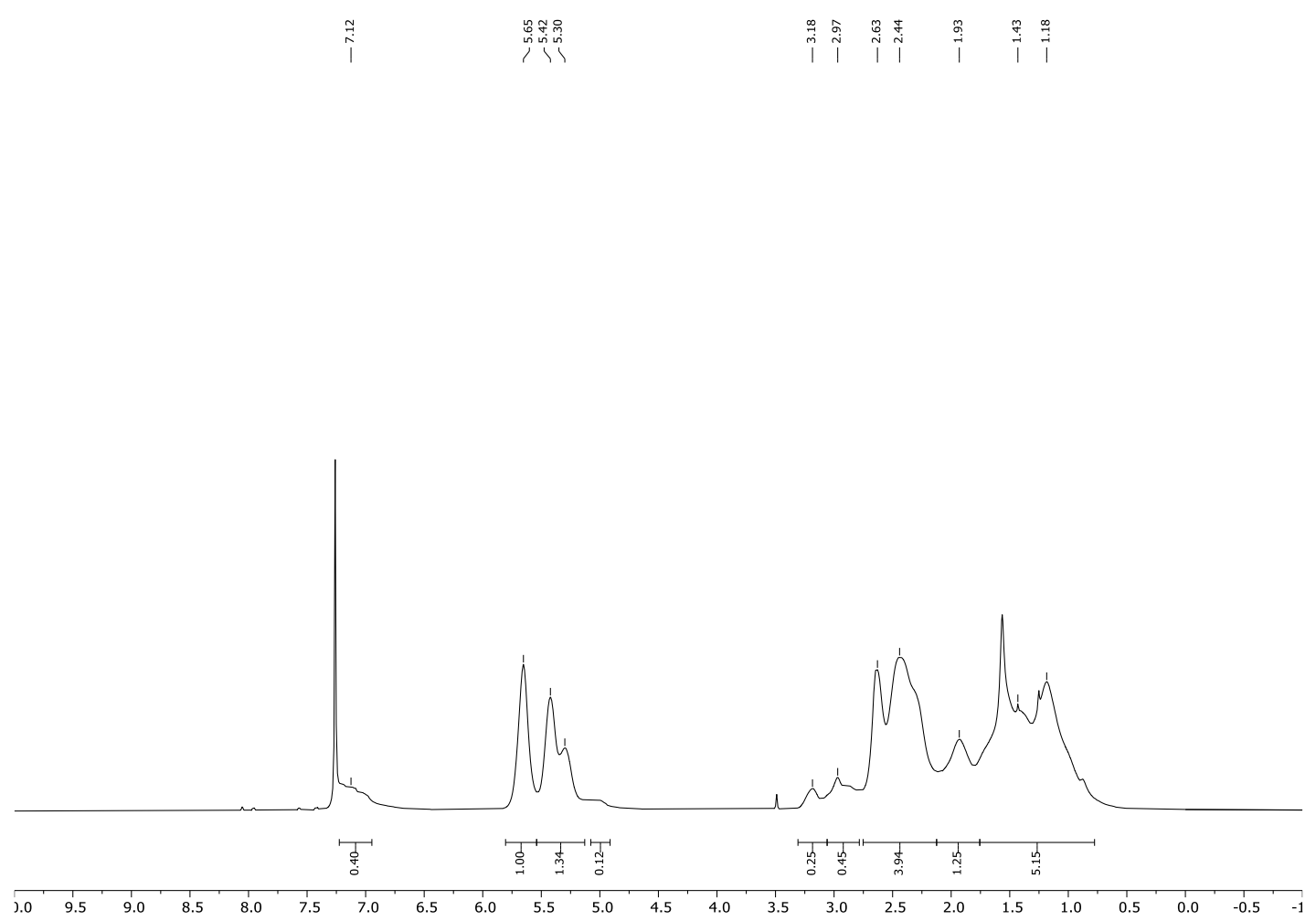

Figure S34. Representative ${ }^{1} \mathrm{H}-\mathrm{NMR}\left(600 \mathrm{MHz}, \mathrm{CDCl}_{3}\right)$ for the epoxidation of $\mathbf{1 2}$ to $\mathbf{1 5 .}$

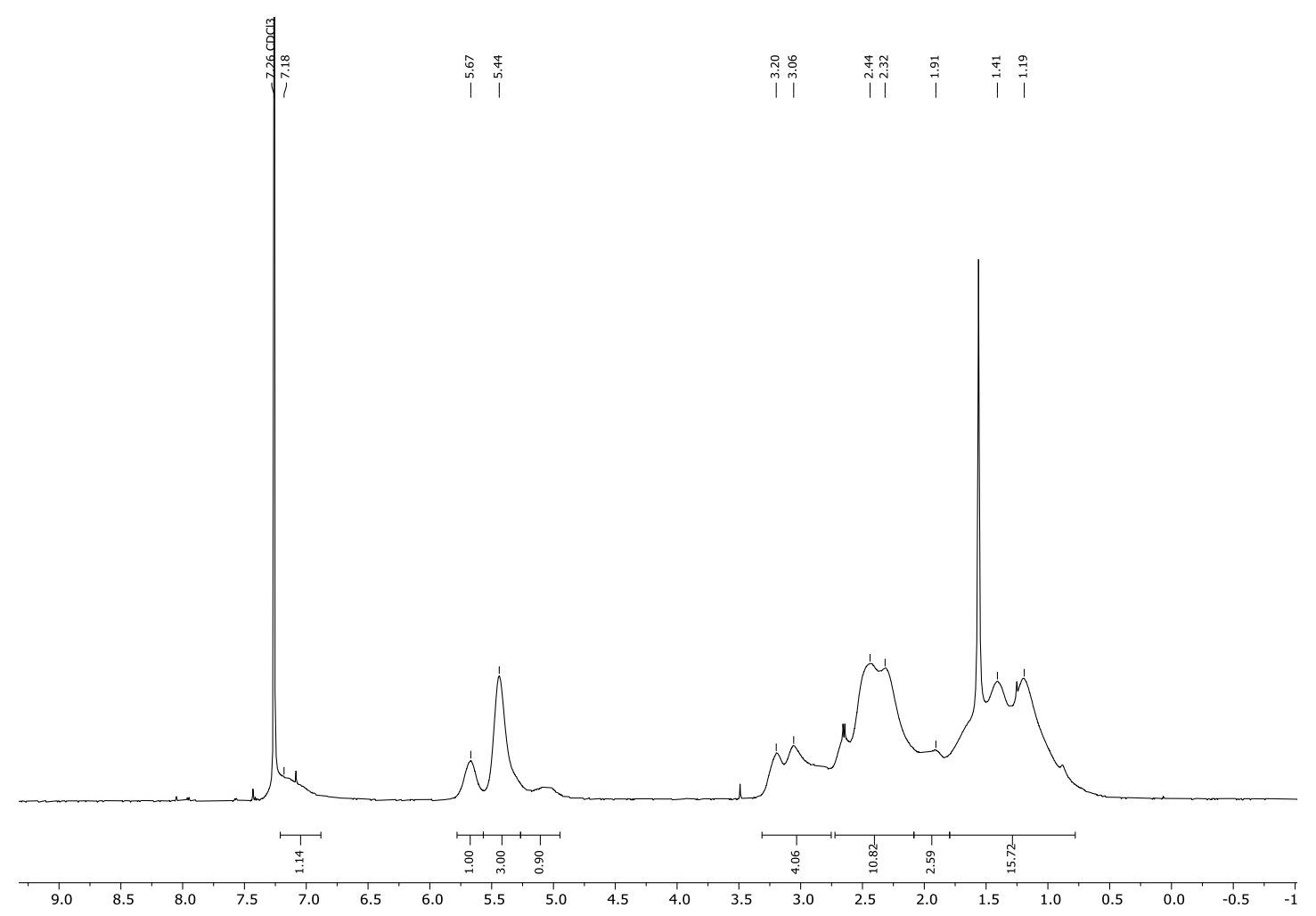

Figure S35. Representative ${ }^{1} \mathrm{H}-\mathrm{NMR}\left(600 \mathrm{MHz}, \mathrm{CDCl}_{3}\right)$ for the epoxidation of $\mathbf{1 2}$ to $\mathbf{1 6 .}$ 


\section{References}

1. Burfield, D. R.; Smithers, R. H. Desiccant Efficiency in Solvent Drying. 3. Dipolar Aprotic Solvents. J. Org. Chem. 1978, 43 (20), 3966-3968.

2. Peters, B. K.; Rodriguez, K. X.; Reisberg, S. H.; Beil, S. B.; Hickey, D. P.; Kawamata, Y.; Collins, M.; Starr, J.; Chen, L.; Udyavara, S.; Klunder, K.; Gorey, T. J.; Anderson, S. L.;

Neurock, M.; Minteer, S. D.; Baran, P. S. Scalable and Safe Synthetic Organic Electroreduction Inspired by Li-lon Battery Chemistry. Science 2019, 363 (6429), 838-845.

3. Walsh, D. J.; Schinski, D. A.; Schneider, R. A.; Guironnet, D. General Route to Design Polymer Molecular Weight Distributions through Flow Chemistry. Nat Commun 2020, 11 (1), 3094. 